\title{
Effects of molecule force on free vibration for a micro electromagnetic harmonic drive system
}

\author{
Dan Zhao ${ }^{1,2}$, Lizhong $\mathrm{Xu}^{1, *}$, and Yuming $\mathrm{Fu}^{1}$ \\ 1 Yanshan University, Mechanical engineering institute, Qinhuangdao 066004, PR China \\ ${ }^{2}$ Hengshui University, Electromechanic research institute, Hengshui 053000, PR China
}

Received: 6 March 2020 / Accepted: 30 January 2021

\begin{abstract}
In this paper, a micro electromagnetic harmonic drive system is proposed. Considering Van der Waals force, dynamics equation of the flexible ring for the micro drive system is deduced and resolved. Using the equations, the effects of the molecule force on the natural frequencies and vibration modes of the drive system are investigated. Results show that considering molecule force, natural frequencies of the flexible ring are reduced and its vibration modes are changed. For lower order modes, smaller clearance between the flexible ring and stator, smaller thickness of the flexible ring and larger radius of the flexible ring, the effects of the molecule force on the natural frequencies and vibration modes are more obvious.
\end{abstract}

Keywords: Electromagnetic harmonic drive system / micro drive / Van der Waals force / free vibration / natural frequency / vibration modes

\section{Introduction}

Electromagnetic harmonic drive is a kind of mechatronics drive device combining rotating magnetic field with harmonic drive. It is widely used in the driving mechanism of machine tools and instruments, and is suitable for submarine navigation, aerospace and transportation fields [1-5].

Herdeg proposed the electromagnetic harmonic drive, and successfully developed the experimental prototype of the drive with external magnetic poles [6]. Janes developed an electromagnetic harmonic drive system with built-in electromagnetic winding, which is compact in structure and can transmit more torque per unit space [7-9]. Rens developed a new type of permanent magnet harmonic drive prototype, which is suitable for the requirements of large transmission ratio [10,11]. Tjahjowidodo proposed a harmonic drive model using statistical measures of variation and analyzed its reliability under different conditions $[12,13]$. Reinhard investigated small harmonic gear drive and developed the harmonic reducer with metal gears which was used in robot driverfor semiconductor chip packaging $[14,15]$. Jose designed harmonic drive with low temperature magnetic superconductor to improve the service life [16]. Chigira proposed a harmonic drive with a stackable structure that is easy to assemble. By adjusting the structure of the magnetic gear, the maximum transfer

\footnotetext{
* e-mail: xlz@ysu.edu.cn
}

torque can be increased [17]. Based on studies of the electromagnetic harmonic drive, $\mathrm{Xu}$ proposed an electromagnetic harmonic movable tooth drive system and investigated its output torque [18-20]. Afanas'evcalculated the electromagnetic moments of anelectromagnetic gear reducer by the energy method [21]. Ando proposed a new harmonic gear with stackable structure easy to assemble and studied the effect of stack on the maximum transmit torque by experiments [22]. Koji proposed a new magnetic harmonic gear which has the stackable structure in which the maximum transmission torque of the gear was improved approximately 5.96 times and the torque density was improved about 3.82 times [23]. Liu et al. investigated eccentric harmonic magnetic gear and presented an analytical method for predicting the distribution of magnetic field in the air gap of harmonic gear [24]. Jing proposed a new type of eccentric harmonic magnetic gear and calculated corresponding magnetic field and static torque with the finite element analysis [25]. With the development of MEMS technology, the size of the driving link is more and more limited, and the micron scale drive technology is urgently needed [26].

Therefore, the Authors propose a micro electromagnetic harmonic drive system. It has advantages such as small volume, light weight, simple and compact structure, large speed ratio and small inertia, etc. Stator components with electrode segments and special piezoelectric ceramic materials are not needed, which is more conducive to the miniaturization. 


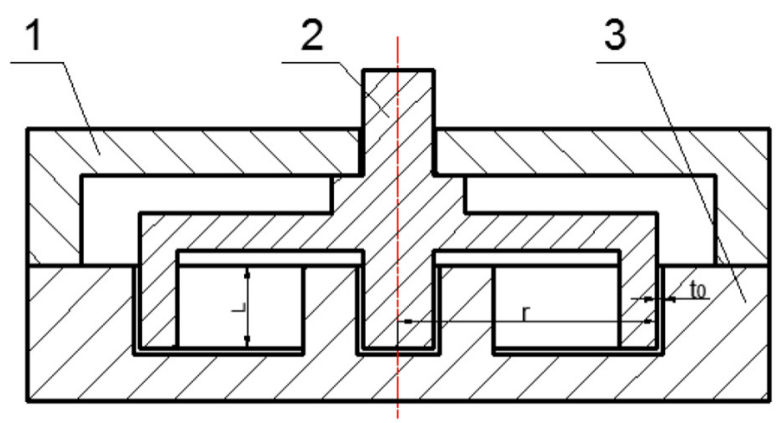

(a)

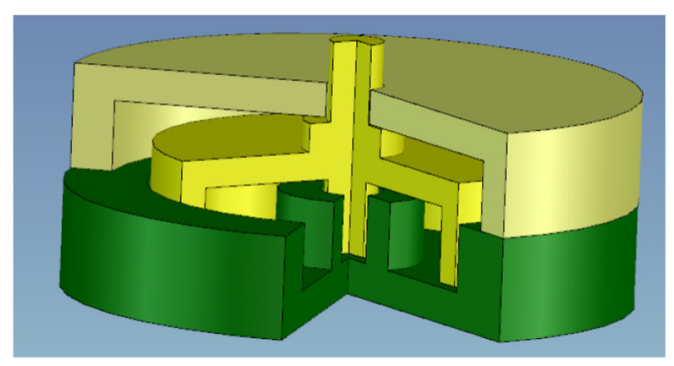

(b)

Fig. 1. An electromagnetic micro harmonic drive system. 1 Cover; 2 flexible ring; 3 stator. (a) Section diagram (b) 3D model.

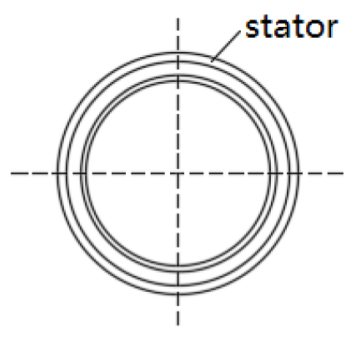

(a)

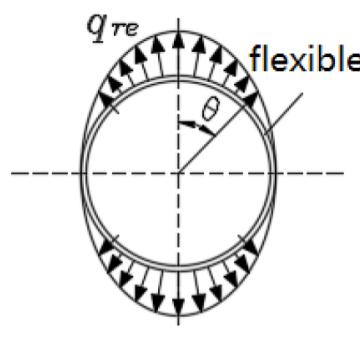

(b)

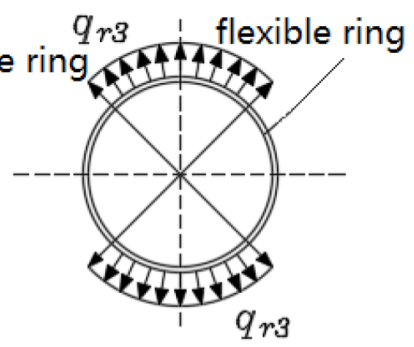

(c)

Fig. 2. Electromagnetic and molecular forces on the flexible ring. (a) Flexible ring and stator (b) electromagnetic force (c) molecular force.

In the drive system, the electromagnetic force has a decisive role for its operation behavior. However, with further reducing the size of the system, the effect of molecular force is becoming more and more significant. The Van der Waals force is an important molecular force, will significantly influence the dynamic performance of the micro electromagnetic harmonic drive system.

In this paper, considering Van der Waals force, dynamics equation of the flexible ring for the micro drive system is deduced and resolved. Using the equations, effects of the molecule force on the natural frequencies and vibration modes of the drive system are investigated. Results show that considering molecule force, natural frequencies of the flexible ring are reduced and its vibration modes are changed. The research is useful in design of the dynamics performance for the micro electromagnetic harmonic drive system.

\section{Structure and operation principle}

Figure 1 shows a micro electromagnetic harmonic drive system. It consists of micro flexible ring and stator. When the external magnetic field is applied sequentially, the rotating magnetic field will cause the flexible wheel to undergo periodic elastic deformation, thus driving the associated supporting shaft to rotate.
Here, $r$ is radius of the flexible ring, $l$ is the length of the flexible ring, $t_{0}$ is the clearance between the flexible ring and stator. The stator material is no magnetic and the flexible ring material is magnetic. The flexible ring under electromagnetic force and molecule force is given in Figure 2. In electromagnetic field, distributed electromagnetic force occurs on the flexible ring. $q_{r e}$ is electromagnetic force per unit length. Meanwhile, molecule force occurs at the angle range $\left[-\frac{\pi}{4}, \frac{\pi}{4}\right] \cdot q_{r 3}$. is Van der Waals force per unit length.

\section{Static displacement of the flexible ring}

The electromagnetic force per unit length on the flexible wheel is

$$
q_{r e}=\frac{n_{B}^{2} \mu_{0} R_{I}^{4} l}{8\left(R_{I}^{2}+x_{B}^{2}\right)^{3}} I^{2} \cos \theta
$$

where $\mu_{0}$-vacuum permeability, $\mu_{0}=4 \pi \times 10^{-7} \mathrm{~N} \cdot \mathrm{A}^{-2}$;

$I$-current in magnetic coil;

$n_{B}$-number of turns per unit length;

$R_{I}$ - magnetic coil radius; 
$x_{B}$-distance from the flexible ring to the magnetic coil plane;

$\theta$-position angle of the flexible ring.

Equation (1) can be simplified to

$$
q_{r e}=q_{r 0} \cos \theta
$$

where $q_{r 0}=\frac{n_{B}^{2} \mu_{0} R_{I}^{4} l}{8\left(R_{I}^{2}+x_{B}^{2}\right)^{3}} I^{2}$

The van der Waals force per unit length is [27]

$$
q_{r 3}=\frac{A l}{6 \pi\left(t_{0}-u_{0}\right)^{3}}
$$

Where $A$ is Hamaker constant, $A=6.58 \times 10^{-20} J$.

The total force per unit length on the flexible ring is

$$
q_{r \mathrm{~s}}=q_{r e}+q_{r 3}
$$
follows:

The distribution of forces on the flexible wheel is as See equation (5) below.
This load is expanded as a Fourier series

$$
q_{r s}=q_{0}+\sum_{1}^{\infty} q_{k} \cos k \theta
$$

The coefficients of the series satisfy the following conditions

$$
\text { See equation (7) below. }
$$

Thus

$$
q_{0}=\frac{2 q_{r 0}}{\pi}+\frac{q_{r 3}}{2}
$$

To determine the coefficient $q_{k}$, multiply the above integral equation by $\cos k \theta$, the following relation is obtained

See equation (9) below.

$$
\begin{aligned}
& \begin{cases}q_{r s}=q_{r 0} \cos \theta+q_{r 3}=\frac{n_{B}^{2} \mu_{0} R_{I}^{4} l}{8\left(R_{I}^{2}+x_{B}^{2}\right)^{3}} I^{2} \cos \theta+q_{r 3} & \left(-\frac{\pi}{4} \leq \theta \leq \frac{\pi}{4}\right) \\
q_{r s}=q_{r 0} \cos \theta & \left(\frac{\pi}{4} \leq \theta \leq \frac{\pi}{2},-\frac{\pi}{2} \leq \theta \leq-\frac{\pi}{4}\right) \\
q_{r s}=-q_{r 0} \cos \theta+q_{r 3}=-\frac{n_{B}^{2} \mu_{0} R_{I}^{4} l}{8\left(R_{I}^{2}+x_{B}^{2}\right)^{3}} I^{2} \cos \theta+q_{r 3} & \left(\frac{3 \pi}{4} \leq \theta \leq \frac{5 \pi}{4}\right) \\
q_{r s}=-q_{r 0} \cos \theta & \left(\frac{\pi}{2} \leq \theta \leq \frac{3 \pi}{4}, \frac{4 \pi}{4} \leq \theta \leq \frac{3 \pi}{2}\right)\end{cases} \\
& \int_{0}^{2 \pi}\left[q_{0}+\sum_{1}^{\infty} q_{k} \cos k \theta\right] d \theta=\int_{-\frac{\pi}{4}}^{\frac{\pi}{4}}\left(q_{r 0} \cos \theta+q_{r 3}\right) d \theta+\int_{\frac{\pi}{4}}^{\frac{\pi}{2}}\left(q_{r 0} \cos \theta\right) d \theta \\
& +\int_{\frac{3 \pi}{4}}^{\frac{5 \pi}{4}}\left(-q_{r 0} \cos \theta+q_{r 3}\right) d \theta+\int_{\frac{\pi}{2}}^{\frac{3 \pi}{4}}\left(-q_{r 0} \cos \theta\right) d \theta \\
& +\int_{\frac{5 \pi}{4}}^{\frac{3 \pi}{2}}\left(-q_{r 0} \cos \theta\right) d \theta+\int_{-\frac{\pi}{2}}^{-\frac{\pi}{4}}\left(q_{r 0} \cos \theta\right) d \theta \\
& \int_{0}^{2 \pi}\left[q_{0}+\sum_{1}^{\infty} q_{k} \cos k \theta\right] \cos k \theta d \theta=\int_{-\frac{\pi}{4}}^{\frac{\pi}{4}}\left(q_{r 0} \cos \theta+q_{r 3}\right) \cos k \theta d \theta+\int_{\frac{\pi}{4}}^{\frac{\pi}{2}}\left(q_{r 0} \cos \theta\right) \cos k \theta d \theta \\
& +\int \frac{\frac{5 \pi}{4}}{4}\left(-q_{r 0} \cos \theta+q_{r 3}\right) \cos k \theta d \theta+\int_{\frac{\pi}{2}}^{\frac{3 \pi}{4}}\left(-q_{r 0} \cos \theta\right) \cos k \theta d \theta \\
& +\int_{\frac{5 \pi}{4}}^{\frac{3 \pi}{2}}\left(-q_{r 0} \cos \theta\right) \cos k \theta d \theta+\int_{-\frac{\pi}{2}}^{-\frac{\pi}{4}}\left(q_{r 0} \cos \theta\right) \cos k \theta d \theta
\end{aligned}
$$


In order to make the load symmetric with respect to the vertical axis and the horizontal axis, only take the coefficient of the cosine series to be even, yields

$q_{k}=\frac{2 q_{r 0}}{\pi}\left[\frac{\sin \frac{(k+1) \pi}{2}}{k+1}+\frac{\sin \frac{(k-1) \pi}{2}}{k-1}\right]+\frac{4 q_{r 3}}{\pi k} \sin \frac{k \pi}{4} \quad(k \neq 1)$

Equation (10) can be changed into following form

$q_{r s}=\frac{2 q_{r 0}}{\pi}+\frac{q_{r 3}}{2}+\sum_{k=2,4,6, \cdots}$

$\times\left\{\frac{2 q_{r 0}}{\pi}\left[\frac{\sin \frac{(k+1) \pi}{2}}{k+1}+\frac{\sin \frac{(k-1) \pi}{2}}{k-1}\right]+\frac{4 q_{r 3}}{\pi k} \sin \frac{k \pi}{4}\right\} \cos k \theta$

Substituting $q_{r s}$ into the dynamics equation of the flexible wheel:

$$
\frac{\partial^{5} u}{\partial \theta^{5}}+2 \frac{\partial^{3} u}{\partial \theta^{3}}+\frac{\partial u}{\partial \theta}=\frac{r^{4}}{E I_{x}} \frac{\partial q_{r s}}{\partial \theta}-\frac{r^{4} \rho A}{E I_{x}} \frac{\partial \ddot{u}}{\partial \theta}
$$

This is a static solution, so the derivative of the displacement with respect to time is zero, that is, $\partial \ddot{u} / \partial \theta=0$, yields

$$
\begin{aligned}
& \frac{\partial^{5} u}{\partial \theta^{5}}+2 \frac{\partial^{3} u}{\partial \theta^{3}}+\frac{\partial u}{\partial \theta}=-\frac{r^{4}}{E I_{x} \pi} \sum_{k=2,4,6, \cdots} \\
& \times\left\{2 q_{r 0}\left[\frac{k \sin \frac{(k+1) \pi}{2}}{k+1}+\frac{k \sin \frac{(k-1) \pi}{2}}{k-1}\right]+4 q_{r 3} \sin \frac{k \pi}{4}\right\} \sin k \theta
\end{aligned}
$$

Write the solution of equation (13) as a series as follows

$$
u=\sum_{k=2,4,6, \cdots} C_{k} \cos k \theta
$$

Substituting equation (14) into equation (13) to obtain

$$
\sum_{k=2,4,6, \cdots} C_{k}\left(-k^{5}+2 k^{3}-k\right) \sin k \theta=-\frac{r^{4}}{E I_{x}} \sum_{k=2,4,6, \cdots}
$$

$$
\times\left\{\frac{2 q_{r 0} k}{\pi}\left[\frac{\sin \frac{(k+1) \pi}{2}}{k+1}+\frac{\sin \frac{(k-1) \pi}{2}}{k-1}\right]+\frac{4 q_{r 3}}{\pi} \sin \frac{k \pi}{4}\right\} \sin k \theta
$$

On both sides of equation (15), if the corresponding terms of the same $k$ value are equal, then

$C_{k}=\frac{r^{4}}{E I_{x}} \frac{\frac{2 q_{r 0} k}{\pi}\left[\frac{\sin \frac{(k+1) \pi}{2}}{k+1}+\frac{\sin \frac{(k-1) \pi}{2}}{k-1}\right]+\frac{4 q_{r n}}{\pi} \sin \frac{k \pi}{4}}{k\left(k^{2}-1\right)^{2}}$

So

See equation (17) below.

Letting $u_{0}$ be equal to the average value of the displacement $u$ at the angle range $\left[-\frac{\pi}{4} \leq \theta \leq \frac{\pi}{4}\right]$, using equation (17), $u_{0}$ can be obtained

See equation (18) below.

$$
\begin{aligned}
u & =\frac{r^{4}}{\pi E I_{x}} \sum_{k=2,4,6, \cdots}\left[2 q_{r 0} \frac{(k-1) \sin \frac{(k+1) \pi}{2}+(k+1) \sin \frac{(k-1) \pi}{2}}{\left(k^{2}-1\right)^{3}}+4 q_{r n} \frac{\sin \frac{k \pi}{4}}{k\left(k^{2}-1\right)^{2}}\right] \cos k \theta \\
u_{0} & =\frac{2}{\pi} \int_{-\frac{\pi}{4}}^{\frac{\pi}{4}} u d \theta \\
& =\frac{8 r^{4}}{\pi^{2} E I_{x}} \sum_{k=2,4,6, \cdots}\left[q_{r 0} \frac{(k-1) \sin \frac{(k+1) \pi}{2}+(k+1) \sin \frac{(k-1) \pi}{2}}{k\left(k^{2}-1\right)^{3}}+2 q_{r n} \frac{\sin \frac{k \pi}{4}}{k^{2}\left(k^{2}-1\right)^{2}}\right] \sin \frac{k \pi}{4}
\end{aligned}
$$




\section{Solution of the dynamics equation for flexible ring}

\subsection{Mode function}

Let the radial displacement $u$ of the flexible ring be composed of static displacement $u_{0}$ and dynamic displacement $\Delta u$

$$
u=u_{0}+\Delta u
$$

The radial load $q_{r}$ is composed of static load $q_{r s}$ and dynamic load $\Delta q_{r}$

$$
q_{r}=q_{r s}+\Delta q_{r}
$$

Substituting equation (20) into equation (12), yields

$$
\frac{\partial^{5} \Delta u}{\partial \theta^{5}}+2 \frac{\partial^{3} \Delta u}{\partial \theta^{3}}+\frac{\partial \Delta u}{\partial \theta}=\frac{r^{4}}{E I_{x}} \frac{\partial \Delta q_{r}}{\partial \theta}-\frac{r^{4} \rho A_{s}}{E I_{x}} \frac{\partial \Delta \ddot{u}}{\partial \theta}
$$

where $\Delta \ddot{u}$ is the second derivative of the dynamic displacement $\Delta u$ with respect to time, $E$ is the elastic modulus of the flexible ring material, $\rho$ is density of the flexible ring, $A_{s}$ is the section area of the flexible ring, $I_{x}$ is the cross section modulus of the flexible ring.

Equation (21) can be changed into following form

$$
\frac{\partial^{4} \Delta u}{\partial \theta^{4}}+2 \frac{\partial^{2} \Delta u}{\partial \theta^{2}}+\Delta u=\frac{r^{4}}{E I_{x}} \Delta q_{r}-\frac{r^{4} \rho A}{E I_{x}} \Delta \ddot{u}
$$

where $\Delta q_{r}$ is the dynamic load, $\Delta q_{r}=\frac{d q_{r}}{d u} \Delta u$, from equations (1) and (3), the dynamic load can be determined by:

- Only considering electromagnetic force:

$$
\Delta q_{r e}=\frac{d q_{r e}}{d u} \Delta u_{e}=0
$$

- Considering electromagnetic force and van der Waals force:

$$
\begin{cases}\Delta q_{r 3}=\frac{d q_{r 3}}{d u} \Delta u_{3}=\frac{A l}{2 \pi\left(t_{0}-u_{0}\right)^{4}} \Delta u_{3} & \left(0 \leq \theta \leq \frac{\pi}{4}\right) \\ \Delta q_{r 3}=0 & \left(\frac{\pi}{4} \leq \theta \leq \frac{\pi}{2}\right)\end{cases}
$$

Substituting equations (23) and (24) into (22), the dynamics equation of the micro electromagnetic harmonic drive system is as follows:
- Only considering electromagnetic force:

$$
\frac{\partial^{4} \Delta u_{e}}{\partial \theta^{4}}+2 \frac{\partial^{2} \Delta u_{e}}{\partial \theta^{2}}+\Delta u_{e}=-\frac{r^{4}}{E I_{x}} \rho A_{s} \Delta \ddot{u}_{e}
$$

- Considering electromagnetic force and van der Waals force:

$$
\text { See equation (26) below. }
$$

Letting

$$
\Delta u=\phi(\theta) q(t)
$$

Then

- Only considering electromagnetic force:

$$
\frac{\ddot{q}(t)}{q(t)}=-\frac{\phi_{e}^{(4)}(\theta)+2 \phi_{e}^{\prime \prime}(\theta)+\phi_{e}(\theta)}{\frac{\rho A_{s} r^{4}}{E I_{x}} \phi_{e}(\theta)}
$$

From equation (28), it is given

$$
\begin{gathered}
\ddot{q}(t)+\omega^{2} q(t)=0 \\
\phi_{e}^{(4)}(\theta)+2 \phi_{e}^{\prime \prime}(\theta)+Q \phi_{e}(\theta)=0
\end{gathered}
$$

where $Q=1-\frac{\rho A_{s} r^{4}}{E I_{x}} \omega^{2}$

Letting $\phi_{e}(\theta)=e^{\lambda \theta}$ and substituting it into (30), yields

$$
\lambda^{4}+2 \lambda^{2}+Q=0
$$

The four eigen values can be obtained as follows $\pm \sqrt{-1+\sqrt{1-Q}}$ and $\pm i \sqrt{1+\sqrt{1-Q}}$, and then the mode function can be given by

$$
\phi_{e}(\theta)=B_{1} \cos k_{1} \theta+B_{2} \sin k_{1} \theta+B_{3} \operatorname{ch} k_{2} \theta+B_{4} \operatorname{sh} k_{2} \theta
$$

where $k_{1}=\sqrt{1+\sqrt{1-Q}}$ and $k_{2}=\sqrt{-1+\sqrt{1-Q}}$

The integral constants $B_{j}(j=1,2,3,4)$ and frequency equation can be determined by symmetry and continuity conditions of the flexible ring.

- Considering electromagnetic force and van der Waals force:

(a) at $0 \leq \theta \leq \frac{\pi}{4}$

In a same manner, following equation can be given

$$
\frac{\ddot{q}(t)}{q(t)}=-\frac{\phi_{31}^{(4)}(\theta)+2 \phi_{31}^{\prime \prime}(\theta)+P \phi_{31}(\theta)}{\frac{\rho A_{s} r^{4}}{E I_{x}} \phi_{31}(\theta)}
$$

where $P=1-\frac{r^{4} A l}{2 E I_{x} \pi\left(t_{0}-u_{0}\right)^{4}}$

$$
\begin{cases}\frac{\partial^{4} \Delta u_{13}}{\partial \theta^{4}}+2 \frac{\partial^{2} \Delta u_{13}}{\partial \theta^{2}}+\Delta u_{13}=\frac{r^{4}}{E I_{x}} \frac{A l}{2 \pi\left(t_{0}-u_{0}\right)^{4}} \Delta u_{13}-\frac{r^{4}}{E I_{x}} \rho A_{s} \Delta \ddot{u}_{13} & \left(0 \leq \theta \leq \frac{\pi}{4}\right) \\ \frac{\partial^{4} \Delta u_{23}}{\partial \theta^{4}}+2 \frac{\partial^{2} \Delta u_{23}}{\partial \theta^{2}}+\Delta u_{23}=-\frac{r^{4}}{E I_{x}} \rho A_{s} \Delta \ddot{u}_{23} & \left(\frac{\pi}{4} \leq \theta \leq \frac{\pi}{2}\right)\end{cases}
$$


From equation (33), it is obtained

$$
\phi_{31}^{(4)}(\theta)+2 \phi_{31}^{\prime \prime}(\theta)+R \phi_{31}(\theta)=0
$$

where $R=P-\frac{\rho A_{s} r^{4}}{E I_{x}} \omega^{2}$

Letting $\phi_{31}(\theta)=e^{\lambda \theta}$ and substituting it into (34), yields

$$
\lambda^{4}+2 \lambda^{2}+R=0
$$

Thus, the mode function can be given as

$$
\begin{aligned}
\phi_{31}(\theta) & =A_{1} \cos m_{1} \theta+A_{2} \sin m_{1} \theta+A_{3} \operatorname{ch} m_{2} \theta \\
& +A_{4} \operatorname{sh} m_{2} \theta
\end{aligned}
$$

where $m_{1}=\sqrt{1+\sqrt{1-R}}, m_{2}=\sqrt{-1+\sqrt{1-R}}$

(b) at $\frac{\pi}{4} \leq \theta \leq \frac{\pi}{2}$

In a same manner, following equation can be given

$$
\frac{\ddot{q}(t)}{q(t)}=-\frac{\phi_{32}^{(4)}(\theta)+2 \phi_{32}^{\prime \prime}(\theta)+\phi_{32}(\theta)}{\frac{\rho A_{s} r^{4}}{E I_{x}} \phi_{32}(\theta)}
$$

From equation (37), it is obtained

$$
\phi_{32}^{(4)}(\theta)+2 \phi_{32}^{\prime \prime}(\theta)+S \phi_{32}(\theta)=0
$$

where $S=1-\frac{\rho A_{s} r^{4}}{E I_{x}} \omega^{2}$

Letting $\phi_{32}(\theta)=e^{\lambda \theta}$ and substituting it into (38), yields

$$
\lambda^{4}+2 \lambda^{2}+S=0
$$

Thus, the mode function can be given as

$$
\phi_{32}(\theta)=A_{5} \cos n_{1} \theta+A_{6} \sin n_{1} \theta+A_{7} \operatorname{chn} n_{2} \theta+A_{8} \operatorname{shn} n_{2} \theta
$$

where $n_{1}=\sqrt{1+\sqrt{1-S}}$ and $n_{2}=\sqrt{-1+\sqrt{1-S}}$.

The integral constants $A_{j}(j=1,2,3,4,5,6,7,8)$ and frequency equation can be determined by symmetry and continuity conditions of the flexible ring.

\subsection{Natural frequencies}

From symmetry and continuity conditions of the flexible ring, both angle $\varphi$ and tangent displacement $v$ are zero at $\theta=0$ and $\theta=\frac{\pi}{2}$, so

$$
\left\{\begin{array}{l}
\phi^{\prime}(\theta)_{\theta=0}=0 \\
\phi^{\prime}(\theta)_{\theta=\frac{\pi}{2}}=0 \\
\phi^{(3)}(\theta)_{\theta=0}=0 \\
\phi^{(3)}(\theta)_{\theta=\frac{\pi}{2}}=0
\end{array}\right.
$$

- Only considering electromagnetic force:

Substituting equation (41) into (32), yields

$$
\text { See equation (42) below. }
$$

From equation (42), it can be known that $B_{2}=0$ and $B_{4}=0$, so equation (42) can be changed into following form

$$
C_{e} X_{e}=D_{e}
$$

where

$$
\begin{gathered}
C_{e}=\left[\begin{array}{cc}
-k_{1} \sin \left(\frac{\pi}{2} k_{1}\right) & k_{2} \operatorname{sh}\left(\frac{\pi}{2} k_{2}\right) \\
k_{1}^{3} \sin \left(\frac{\pi}{2} k_{1}\right) & k_{2}^{3} \operatorname{sh}\left(\frac{\pi}{2} k_{2}\right)
\end{array}\right] \\
X_{e}=\left[\begin{array}{ll}
B_{1} & B_{3}
\end{array}\right]^{T} D_{e}=\left[\begin{array}{ll}
0 & 0
\end{array}\right]^{T}
\end{gathered}
$$

If there are non-zero solutions for equation (43), the determinant of is zero, that is

$$
\left|\begin{array}{cc}
-k_{1} \sin \left(\frac{\pi}{2} k_{1}\right) & k_{2} \operatorname{sh}\left(\frac{\pi}{2} k_{2}\right) \\
k_{1}^{3} \sin \left(\frac{\pi}{2} k_{1}\right) & k_{2}^{3} \operatorname{sh}\left(\frac{\pi}{2} k_{2}\right)
\end{array}\right|=0
$$

- Considering electromagnetic force and van der Waals force:

From continuity conditions of the flexible ring, following relationship can be given at $\theta=\frac{\pi}{4}$

$$
\left\{\begin{array}{l}
\phi_{31}(\theta)=\phi_{32}(\theta) \\
\phi_{31}^{\prime}(\theta)=\phi_{32}^{\prime}(\theta) \\
\phi_{31}^{\prime \prime}(\theta)=\phi_{32}^{\prime \prime}(\theta) \\
\phi_{31}^{(3)}(\theta)=\phi_{32}^{(3)}(\theta)
\end{array}\right.
$$

$$
\left.\begin{array}{c}
B_{2} k_{1}+B_{4} k_{2}=0 \\
-B_{1} k_{1} \sin \left(\frac{\pi}{2} k_{1}\right)+B_{2} k_{1} \cos \left(\frac{\pi}{2} k_{1}\right)+B_{3} k_{2} \operatorname{sh}\left(\frac{\pi}{2} k_{2}\right)+B_{4} k_{2} \operatorname{ch}\left(\frac{\pi}{2} k_{2}\right)=0 \\
-B_{2} k_{1}^{3}+B_{4} k_{2}^{3}=0 \\
B_{1} k_{1}^{3} \sin \left(\frac{\pi}{2} k_{1}\right)-B_{2} k_{1}^{3} \cos \left(\frac{\pi}{2} k_{1}\right)+B_{3} k_{2}^{3} \operatorname{sh}\left(\frac{\pi}{2} k_{2}\right)+B_{4} k_{2}^{3} \operatorname{ch}\left(\frac{\pi}{2} k_{2}\right)=0
\end{array}\right\}
$$


Substituting equations (36) and (40) into (45), yields following form

See equation (46) below.

From equation (46), it can be known that $A_{2}=0$ and $A_{4}=0$, so equation (46) can be changed into

$$
C_{1} X_{1}=D_{1}
$$

where

\begin{abstract}
$A_{4}=0$, so equation $(46)$ can be changed into
\end{abstract} .

$$
\begin{gathered}
A_{2} m_{1}+A_{4} m_{2}=0 \\
-A_{5} n_{1} \sin \left(\frac{\pi}{2} n_{1}\right)+A_{6} n_{1} \cos \left(\frac{\pi}{2} n_{1}\right)+A_{7} n_{2} \operatorname{sh}\left(\frac{\pi}{2} n_{2}\right)+A_{8} n_{2} \operatorname{ch}\left(\frac{\pi}{2} n_{2}\right)=0 \\
-A_{2} m_{1}^{3}+A_{4} m_{2}^{3}=0 \\
A_{5} n_{1}^{3} \sin \left(\frac{\pi}{2} n_{1}\right)-A_{6} n_{1}^{3} \cos \left(\frac{\pi}{2} n_{1}\right)+A_{7} n_{2}^{3} \operatorname{sh}\left(\frac{\pi}{2} n_{2}\right)+A_{8} n_{2}^{3} \operatorname{ch}\left(\frac{\pi}{2} n_{2}\right)=0 \\
A_{1} \cos \left(\frac{\pi}{4} m_{1}\right)+A_{2} \sin \left(\frac{\pi}{4} m_{1}\right)+A_{3} \operatorname{ch}\left(\frac{\pi}{4} m_{2}\right)+A_{4} \operatorname{sh}\left(\frac{\pi}{4} m_{2}\right)-A_{5} \cos \left(\frac{\pi}{4} n_{1}\right) \\
-A_{6} \sin \left(\frac{\pi}{4} n_{1}\right)-A_{7} \operatorname{ch}\left(\frac{\pi}{4} n_{2}\right)-A_{8} \operatorname{sh}\left(\frac{\pi}{4} n_{2}\right)=0 \\
-A_{1} m_{1} \sin \left(\frac{\pi}{4} m_{1}\right)+A_{2} m_{1} \cos \left(\frac{\pi}{4} m_{1}\right)+A_{3} m_{2} \operatorname{sh}\left(\frac{\pi}{4} m_{2}\right)+A_{4} m_{2} \operatorname{ch}\left(\frac{\pi}{4} m_{2}\right) \\
+A_{5} n_{1} \sin \left(\frac{\pi}{4} n_{1}\right)-A_{6} n_{1} \cos \left(\frac{\pi}{4} n_{1}\right)-A_{7} n_{2} \operatorname{sh}\left(\frac{\pi}{4} n_{2}\right)-A_{8} n_{2} \operatorname{ch}\left(\frac{\pi}{4} n_{2}\right)=0 \\
-A_{1} m_{1}^{2} \cos \left(\frac{\pi}{4} m_{1}\right)-A_{2} m_{1}^{2} \sin \left(\frac{\pi}{4} m_{1}\right)+A_{3} m_{2}^{2} \operatorname{ch}\left(\frac{\pi}{4} m_{2}\right)+A_{4} m_{2}^{2} \operatorname{sh}\left(\frac{\pi}{4} m_{2}\right) \\
+A_{5} n_{1}^{2} \cos \left(\frac{\pi}{4} n_{1}\right)+A_{6} n_{1}^{2} \sin \left(\frac{\pi}{4} n_{1}\right)-A_{7} n_{2}^{2} \operatorname{ch}\left(\frac{\pi}{4} n_{2}\right)-A_{8} n_{2}^{2} \operatorname{sh}\left(\frac{\pi}{4} n_{2}\right)=0 \\
A_{1} m_{1}^{3} \sin \left(\frac{\pi}{4} m_{1}\right)-A_{2} m_{1}^{3} \cos \left(\frac{\pi}{4} m_{1}\right)+A_{3} m_{2}^{3} \operatorname{sh}\left(\frac{\pi}{4} m_{2}\right)+A_{4} m_{2}^{3} \operatorname{ch}\left(\frac{\pi}{4} m_{2}\right) \\
-A_{5} n_{1}^{3} \sin \left(\frac{\pi}{4} n_{1}\right)+A_{6} n_{1}^{3} \cos \left(\frac{\pi}{4} n_{1}\right)-A_{7} n_{2}^{3} \operatorname{sh}\left(\frac{\pi}{4} n_{2}\right)-A_{8} n_{2}^{3} \operatorname{ch}\left(\frac{\pi}{4} n_{2}\right)=0
\end{gathered}
$$

$$
C_{1}=\left[\begin{array}{cccccc}
0 & 0 & -n_{1} \sin \left(\frac{\pi}{2} n_{1}\right) & n_{1} \cos \left(\frac{\pi}{2} n_{1}\right) & n_{2} \operatorname{sh}\left(\frac{\pi}{2} n_{2}\right) & n_{2} \operatorname{ch}\left(\frac{\pi}{2} n_{2}\right) \\
0 & 0 & n_{1}^{3} \sin \left(\frac{\pi}{2} n_{1}\right) & -n_{1}^{3} \cos \left(\frac{\pi}{2} n_{1}\right) & n_{2}^{3} \operatorname{sh}\left(\frac{\pi}{2} n_{2}\right) & n_{2}^{3} \operatorname{ch}\left(\frac{\pi}{2} n_{2}\right) \\
\cos \left(\frac{\pi}{4} m_{1}\right) & \operatorname{ch}\left(\frac{\pi}{4} m_{2}\right) & -\cos \left(\frac{\pi}{4} n_{1}\right) & -\sin \left(\frac{\pi}{4} n_{1}\right) & -\operatorname{ch}\left(\frac{\pi}{4} n_{2}\right) & -\operatorname{sh}\left(\frac{\pi}{4} n_{2}\right) \\
-m_{1} \sin \left(\frac{\pi}{4} m_{1}\right) & m_{2} \operatorname{sh}\left(\frac{\pi}{4} m_{2}\right) & n_{1} \sin \left(\frac{\pi}{4} n_{1}\right) & -n_{1} \cos \left(\frac{\pi}{4} n_{1}\right) & -n_{2} \operatorname{sh}\left(\frac{\pi}{4} n_{2}\right) & -n_{2} \operatorname{ch}\left(\frac{\pi}{4} n_{2}\right) \\
-m_{1}^{2} \cos \left(\frac{\pi}{4} m_{1}\right) & m_{2}^{2} \operatorname{ch}\left(\frac{\pi}{4} m_{2}\right) & n_{1}^{2} \cos \left(\frac{\pi}{4} n_{1}\right) & n_{1}^{2} \sin \left(\frac{\pi}{4} n_{1}\right) & -n_{2}^{2} \operatorname{ch}\left(\frac{\pi}{4} n_{2}\right) & -n_{2}^{2} \operatorname{sh}\left(\frac{\pi}{4} n_{2}\right) \\
m_{1}^{3} \sin \left(\frac{\pi}{4} m_{1}\right) & m_{2}^{3} \operatorname{sh}\left(\frac{\pi}{4} m_{2}\right) & -n_{1}^{3} \sin \left(\frac{\pi}{4} n_{1}\right) & n_{1}^{3} \cos \left(\frac{\pi}{4} n_{1}\right) & -n_{2}^{3} \operatorname{sh}\left(\frac{\pi}{4} n_{2}\right) & -n_{2}^{3} \operatorname{ch}\left(\frac{\pi}{4} n_{2}\right)
\end{array}\right]
$$


If there are non-zero solutions for equation (47), the corresponding determinant is zero, that is

$$
\text { See equation (48) below. }
$$

Using equations (42) and (46), the constants $B_{j}$ and $A_{j}$ of mode functions can be determined. Using equation. (44) and (48), natural frequencies of the flexible ring can be determined. Then, the primary mass $M_{p i}$ of the first order can be calculated by

$$
M_{p i}=\int_{0}^{\beta} \rho\left[\phi_{1 i}(\theta)\right]^{2} A r d \theta+\int_{\beta}^{\pi / 2} \rho\left[\phi_{2 i}(\theta)\right]^{2} A r d \theta
$$

Multiplying $\phi_{i}(\theta)$ by $M_{p i}^{-1 / 2}$, the normal mode function of the flexible ring is obtained.

\section{Effects of friction and air damping}

Under the electromagnetic force, the flexible wheel after deformation contacts with the outer ring stator at the angle range $[-\pi / 4, \pi / 4]$. Here, the friction force occurs. At other angle range, the air damping force is applied to the flexible wheel. Letting $q_{t}$ denote friction force per unit length on the flexible wheel, $q_{p}$ denote damping force per unit length on the flexible wheel.

From friction torque equation of the flexible wheel, the friction force $q_{\mathrm{t} 3}$ considering electromagnetic force and Van der Walls force can be given by

$$
\begin{aligned}
q_{t 3} & =\frac{\mu\left(q_{r 0} Y_{1}+2 q_{r 3} Y_{2}\right)}{2 Y_{2}}-\frac{\mu t_{0} \pi E I_{x}}{4 r^{4} Y_{2}} \\
& =\frac{\mu q_{r 0} Y_{1}}{2 Y_{2}}-\frac{\mu t_{0} \pi E I_{x}}{4 r^{4} Y_{2}}+\mu q_{r 3}
\end{aligned}
$$

This friction force is expanded as a Fourier series and high order terms are neglected, the dynamic friction force can be obtained

$$
\Delta q_{t 3}=\frac{\mu A l}{2 \pi\left(t_{0}-u_{0}\right)^{4}} \Delta u
$$

The air damping force $q_{p}$ per unit length on the flexible wheel is [28]

$$
q_{p}=\frac{\eta l^{3}}{\left(t_{0}-u\right)^{3}} \frac{\partial u}{\partial t}
$$

where $\eta$ is the air dynamic viscosity, $\eta=1.86 \times 10^{-5}$ $\mathrm{N} \cdot \mathrm{S} \cdot \mathrm{m}^{-2}$.

This air damping force is expanded as a Fourier series and high order terms are neglected, the dynamic air damping force can be obtained

$$
q_{p}=\frac{\eta l^{3}}{\left(t_{0}-u_{0}\right)^{3}} \frac{\partial \Delta u}{\partial t}
$$

Considering tangent friction force, the dynamics equation (12) of the flexible wheel is changed into following form

$$
\frac{\partial^{5} \Delta u}{\partial \theta^{5}}+2 \frac{\partial^{3} \Delta u}{\partial \theta^{3}}+\frac{\partial \Delta u}{\partial \theta}=\frac{r^{4}}{E I_{x}}\left(\frac{\partial \Delta q_{r}}{\partial \theta}+\Delta q_{t}\right)-\frac{r^{4} \rho A_{s}}{E I_{x}} \frac{\partial \Delta \ddot{u}}{\partial \theta}
$$

The forces on the flexible wheel include radial one and tangent one, and the force distribution can be given by

See equation (55) below.

$$
\begin{array}{|cccccc}
0 & 0 & -n_{1} \sin \left(\frac{\pi}{2} n_{1}\right) & n_{1} \cos \left(\frac{\pi}{2} n_{1}\right) & n_{2} \operatorname{sh}\left(\frac{\pi}{2} n_{2}\right) & n_{2} \operatorname{ch}\left(\frac{\pi}{2} n_{2}\right) \\
0 & 0 & n_{1}^{3} \sin \left(\frac{\pi}{2} n_{1}\right) & -n_{1}^{3} \cos \left(\frac{\pi}{2} n_{1}\right) & n_{2}^{3} \operatorname{sh}\left(\frac{\pi}{2} n_{2}\right) & n_{2}^{3} \operatorname{ch}\left(\frac{\pi}{2} n_{2}\right) \\
\cos \left(\frac{\pi}{4} m_{1}\right) & \operatorname{ch}\left(\frac{\pi}{4} m_{2}\right) & -\cos \left(\frac{\pi}{4} n_{1}\right) & -\sin \left(\frac{\pi}{4} n_{1}\right) & -\operatorname{ch}\left(\frac{\pi}{4} n_{2}\right) & -\operatorname{sh}\left(\frac{\pi}{4} n_{2}\right) \\
-m_{1} \sin \left(\frac{\pi}{4} m_{1}\right) & m_{2} \operatorname{sh}\left(\frac{\pi}{4} m_{2}\right) & n_{1} \sin \left(\frac{\pi}{4} n_{1}\right) & -n_{1} \cos \left(\frac{\pi}{4} n_{1}\right) & -n_{2} \operatorname{sh}\left(\frac{\pi}{4} n_{2}\right) & -n_{2} \operatorname{ch}\left(\frac{\pi}{4} n_{2}\right) \\
-m_{1}^{2} \cos \left(\frac{\pi}{4} m_{1}\right) & m_{2}^{2} \operatorname{ch}\left(\frac{\pi}{4} m_{2}\right) & n_{1}^{2} \cos \left(\frac{\pi}{4} n_{1}\right) & n_{1}^{2} \sin \left(\frac{\pi}{4} n_{1}\right) & -n_{2}^{2} \operatorname{ch}\left(\frac{\pi}{4} n_{2}\right) & -n_{2}^{2} \operatorname{sh}\left(\frac{\pi}{4} n_{2}\right) \\
m_{1}^{3} \sin \left(\frac{\pi}{4} m_{1}\right) & m_{2}^{3} \operatorname{sh}\left(\frac{\pi}{4} m_{2}\right) & -n_{1}^{3} \sin \left(\frac{\pi}{4} n_{1}\right) & n_{1}^{3} \cos \left(\frac{\pi}{4} n_{1}\right) & -n_{2}^{3} \operatorname{sh}\left(\frac{\pi}{4} n_{2}\right) & -n_{2}^{3} \operatorname{ch}\left(\frac{\pi}{4} n_{2}\right)
\end{array} \mid=0
$$


Combining equation (54) with (55), yields

See equation (56) below.

Letting

$$
\Delta u=\phi(\theta) q(t)
$$

Substituting equation (57) into (56), yields:

See equation (58-a) and (58-b) below.

where $T=\frac{r^{4}}{E I_{x}} \frac{A l}{2 \pi\left(t_{0}-u_{0}\right)^{4}}$

From equation (58-a), we know

$$
\phi_{p 1}^{(5)}(\theta)+2 \phi_{p 1}^{(3)}(\theta)+U \phi_{p 1}^{\prime}(\theta)-\mu T \phi_{p 1}(\theta)=0
$$

where $U=1-T-\frac{\rho A_{s} r^{4}}{E I_{x}} \omega^{2}$

Letting $\phi_{p 1}(\theta)=e^{\lambda \theta}$ and substituting it into (59), yields

$$
\lambda^{5}+2 \lambda^{3}+U \lambda-\mu T=0
$$

The five eigenvalues can be obtained $\left(r_{1}, r_{2} \pm i r_{3}\right.$ and $r_{4} \pm i r_{5}$ ), and then the mode function can be given by

$$
\begin{aligned}
\phi_{p 1}(\theta)= & C_{1} e^{r_{1} \theta}+e^{r_{2} \theta}\left(C_{2} \cos r_{3} \theta+C_{3} \sin r_{3} \theta\right) \\
& +e^{r_{4} \theta}\left(C_{4} \cos r_{5} \theta+C_{5} \sin r_{5} \theta\right)
\end{aligned}
$$

From equation (58-b), we know

$$
\phi_{p 2}^{(4)}(\theta)+2 \phi_{p 2}^{\prime \prime}(\theta)+V \phi_{p 2}(\theta)=0
$$

where $V=1-\frac{\rho A_{s} r^{4}}{E I_{x}} \omega^{2}$

Letting $\phi_{p 2}(\theta)=e^{\lambda \theta}$ and substituting it into (62), yields

$$
\lambda^{4}+2 \lambda^{2}+V=0
$$

The four eigenvalues can be obtained $( \pm \sqrt{-1+\sqrt{1-V}}$ and $\pm i \sqrt{1+\sqrt{1-V}})$, and then the mode function can be given by

$$
\phi_{p 2}(\theta)=C_{6} \cos \alpha_{1} \theta+C_{7} \sin \alpha_{1} \theta+C_{8} \operatorname{ch} \alpha_{2} \theta+C_{9} \operatorname{sh} \alpha_{2} \theta
$$

where $\alpha_{1}=\sqrt{1+\sqrt{1-V}}$ and $\alpha_{2}=\sqrt{-1+\sqrt{1-V}}$

From symmetry and continuity conditions of the flexible ring, both angle $\varphi$, tangent displacement $v$ and shear force are zero at $\theta=0$ and $\theta=\frac{\pi}{2}$, so

$$
\left\{\begin{array}{l}
\phi_{p 1}^{\prime}(\theta)_{\theta=0}=0 \\
\phi_{p 2}^{\prime}(\theta)_{\theta=\frac{\pi}{2}}=0 \\
\phi_{p 1}^{(3)}(\theta)_{\theta=0}=0 \\
\phi_{p 2}^{(3)}(\theta)_{\theta=\frac{\pi}{2}}=0
\end{array}\right.
$$

From continuity conditions of the flexible ring, following relationship can be given at $\theta=\frac{\pi}{4}$

$$
\left\{\begin{array}{l}
\phi_{p 1}(\theta)=\phi_{p 2}(\theta) \\
\phi_{p 1}^{\prime}(\theta)=\phi_{p 2}^{\prime}(\theta) \\
\phi_{p 1}^{\prime \prime}(\theta)=\phi_{p 2}^{\prime \prime}(\theta) \\
\phi_{p 1}^{(3)}(\theta)=\phi_{p 2}^{(3)}(\theta) \\
\phi_{p 1}^{(4)}(\theta)=\phi_{p 2}^{(4)}(\theta)
\end{array}\right.
$$

$$
\left\{\begin{array}{l}
\frac{\partial^{5} \Delta u_{1 p}}{\partial \theta^{5}}+2 \frac{\partial^{3} \Delta u_{1 p}}{\partial \theta^{3}}+\frac{\partial \Delta u_{1 p}}{\partial \theta} \\
=\frac{r^{4}}{E I_{x}}\left(\frac{A l}{2 \pi\left(t_{0}-u_{0}\right)^{4}} \frac{\partial \Delta u_{1 p}}{\partial \theta}+\frac{\mu A l}{2 \pi\left(t_{0}-u_{0}\right)^{4}} \Delta u_{1 p}\right)-\frac{r^{4} \rho A_{s}}{E I_{x}} \frac{\partial \Delta \ddot{u}_{1 p}}{\partial \theta} \quad\left(0 \leq \theta \leq \frac{\pi}{4}\right) \\
\frac{\partial^{4} \Delta u_{2 p}}{\partial \theta^{4}}+2 \frac{\partial^{2} \Delta u_{2 p}}{\partial \theta^{2}}+\Delta u_{2 p}=-\frac{r^{4}}{E I_{x}} \frac{\eta l^{3}}{\left(t_{0}-u_{0}\right)^{3}} \frac{\partial \Delta u_{2 p}}{\partial t}-\frac{r^{4}}{E I_{x}} \rho A_{s} \Delta \ddot{u}_{2 p} \quad\left(\frac{\pi}{4} \leq \theta \leq \frac{\pi}{2}\right)
\end{array}\right.
$$

$$
\begin{aligned}
& \frac{\ddot{q}(t)}{q(t)}=-\frac{\phi_{p 1}^{(5)}(\theta)+2 \phi_{p 1}^{(3)}(\theta)+(1-T) \phi_{p 1}^{\prime}(\theta)-\mu T \phi_{p 1}(\theta)}{\frac{\rho A_{s} r^{4}}{E I_{x}} \phi_{p 1}^{\prime}(\theta)} \quad\left(0 \leq \theta \leq \frac{\pi}{4}\right) \\
& \frac{\ddot{q}(t)}{q(t)}+\frac{\eta l^{3}}{\left(t_{0}-u_{0}\right)^{3} \rho A_{s}} \frac{\dot{q}(t)}{q(t)}=-\frac{\phi_{p 2}^{(4)}(\theta)+2 \phi_{p 2}^{\prime \prime}(\theta)+\phi_{p 2}(\theta)}{\frac{\rho A_{s} r^{4}}{E I_{x}} \phi_{p 2}(\theta)} \quad\left(\frac{\pi}{4} \leq \theta \leq \frac{\pi}{2}\right)
\end{aligned}
$$


Substituting equations (61) and (64) into (65) and (66), yields
Equation (67) can be changed into following form

$$
C X_{1}=D_{1}
$$

$$
\begin{aligned}
& C_{1} r_{1}+C_{2} r_{2}+C_{3} r_{3}+C_{4} r_{4}+C_{5} r_{5}=0 \\
& -C_{6} \alpha_{1} \sin \left(\frac{\pi}{2} \alpha_{1}\right)+C_{7} \alpha_{1} \cos \left(\frac{\pi}{2} \alpha_{1}\right)+C_{8} \alpha_{2} \operatorname{sh}\left(\frac{\pi}{2} \alpha_{2}\right)+C_{9} \alpha_{2} \operatorname{ch}\left(\frac{\pi}{2} \alpha_{2}\right)=0 \\
& C_{1} r_{1}^{3}+C_{2}\left(r_{2}^{3}-3 r_{2} r_{3}^{2}\right)+C_{3}\left(3 r_{2}^{2} r_{3}-r_{3}^{3}\right)+C_{4}\left(r_{4}^{3}-3 r_{4} r_{5}^{2}\right)+C_{5}\left(3 r_{4}^{2} r_{5}-r_{5}^{3}\right)=0 \\
& C_{6} \alpha_{1}^{3} \sin \left(\frac{\pi}{2} \alpha_{1}\right)-C_{7} \alpha_{1}^{3} \cos \left(\frac{\pi}{2} \alpha_{1}\right)+C_{8} \alpha_{2}^{3} \operatorname{sh}\left(\frac{\pi}{2} \alpha_{2}\right)+C_{9} \alpha_{2}^{3} \operatorname{ch}\left(\frac{\pi}{2} \alpha_{2}\right)=0 \\
& C_{1} e^{\frac{\pi}{4} r_{1}}+C_{2} e^{\frac{\pi}{4} r_{2}} \cos \left(\frac{\pi}{4} r_{3}\right)+C_{3} e^{\frac{\pi}{4} r_{2}} \sin \left(\frac{\pi}{4} r_{3}\right)+C_{4} e^{\frac{\pi}{4} r_{4}} \cos \left(\frac{\pi}{4} r_{5}\right)+C_{5} e^{\frac{\pi}{4} r_{4}} \sin \left(\frac{\pi}{4} r_{5}\right) \\
& -C_{6} \cos \left(\frac{\pi}{4} \alpha_{1}\right)-C_{7} \sin \left(\frac{\pi}{4} \alpha_{1}\right)-C_{8} \operatorname{ch}\left(\frac{\pi}{4} \alpha_{2}\right)-C_{9} \operatorname{sh}\left(\frac{\pi}{4} \alpha_{2}\right)=0 \\
& C_{1} r_{1} e^{\frac{\pi}{4} r_{1}}+C_{2} e^{\frac{\pi}{4} r_{2}}\left(r_{2} \cos \left(\frac{\pi}{4} r_{3}\right)-r_{3} \sin \left(\frac{\pi}{4} r_{3}\right)\right)+C_{3} e^{\frac{\pi}{4} r_{2}}\left(r_{2} \sin \left(\frac{\pi}{4} r_{3}\right)+r_{3} \cos \left(\frac{\pi}{4} r_{3}\right)\right) \\
& +C_{4} e^{\frac{\pi}{4}} r_{4}\left(r_{4} \cos \left(\frac{\pi}{4} r_{5}\right)-r_{5} \sin \left(\frac{\pi}{4} r_{5}\right)\right)+C_{5} e^{\frac{\pi}{4} r_{4}}\left(r_{4} \sin \left(\frac{\pi}{4} r_{5}\right)+r_{5} \cos \left(\frac{\pi}{4} r_{5}\right)\right) \\
& +C_{6} \alpha_{1} \sin \left(\frac{\pi}{4} \alpha_{1}\right)-C_{7} \alpha_{1} \cos \left(\frac{\pi}{4} \alpha_{1}\right)-C_{8} \alpha_{2} \operatorname{sh}\left(\frac{\pi}{4} \alpha_{2}\right)-C_{9} \alpha_{2} \operatorname{ch}\left(\frac{\pi}{4} \alpha_{2}\right)=0 \\
& C_{1} r_{1}^{2} e^{\frac{\pi}{4} r_{1}}+C_{2} e^{\frac{\pi}{4}} r_{2}\left(\left(r_{2}^{2}-r_{3}^{2}\right) \cos \left(\frac{\pi}{4} r_{3}\right)-2 r_{2} r_{3} \sin \left(\frac{\pi}{4} r_{3}\right)\right) \\
& +C_{3} e^{\frac{\pi}{4}} r_{2}\left(\left(r_{2}^{2}-r_{3}^{2}\right) \sin \left(\frac{\pi}{4} r_{3}\right)+2 r_{2} r_{3} \cos \left(\frac{\pi}{4} r_{3}\right)\right) \\
& +C_{4} e^{\frac{\pi}{4}} r_{4}\left(\left(r_{4}^{2}-r_{5}^{2}\right) \cos \left(\frac{\pi}{4} r_{5}\right)-2 r_{4} r_{5} \sin \left(\frac{\pi}{4} r_{5}\right)\right) \\
& +C_{5} e^{\frac{\pi}{4}} r_{4}\left(\left(r_{4}^{2}-r_{5}^{2}\right) \sin \left(\frac{\pi}{4} r_{5}\right)+2 r_{4} r_{5} \cos \left(\frac{\pi}{4} r_{5}\right)\right) \\
& +C_{6} \alpha_{1}^{2} \cos \left(\frac{\pi}{4} \alpha_{1}\right)+C_{7} \alpha_{1}^{2} \sin \left(\frac{\pi}{4} \alpha_{1}\right)-C_{8} \alpha_{2}^{2} \operatorname{ch}\left(\frac{\pi}{4} \alpha_{2}\right)-C_{9} \alpha_{2}^{2} \operatorname{sh}\left(\frac{\pi}{4} \alpha_{2}\right)=0 \\
& C_{1} r_{1}^{3} e^{\frac{\pi}{4} r_{1}}+C_{2} e^{\frac{\pi}{4}} r_{2}\left(\left(r_{3}^{3}-3 r_{2}^{2} r_{3}\right) \sin \left(\frac{\pi}{4} r_{3}\right)+\left(r_{2}^{3}-3 r_{2} r_{3}^{2}\right) \cos \left(\frac{\pi}{4} r_{3}\right)\right) \\
& +C_{3} e^{\frac{\pi}{4} r_{2}}\left(\left(r_{2}^{3}-3 r_{2} r_{3}^{2}\right) \sin \left(\frac{\pi}{4} r_{3}\right)+\left(3 r_{2}^{2} r_{3}-r_{3}^{3}\right) \cos \left(\frac{\pi}{4} r_{3}\right)\right) \\
& +C_{4} e^{\frac{\pi}{4} r_{4}}\left(\left(r_{5}^{3}-3 r_{4}^{2} r_{5}\right) \sin \left(\frac{\pi}{4} r_{5}\right)+\left(r_{4}^{3}-3 r_{4} r_{5}^{2}\right) \cos \left(\frac{\pi}{4} r_{5}\right)\right) \\
& +C_{5} e^{\frac{\pi}{4} r_{4}}\left(\left(r_{4}^{3}-3 r_{4} r_{5}^{2}\right) \sin \left(\frac{\pi}{4} r_{5}\right)+\left(3 r_{4}^{2} r_{5}-r_{5}^{3}\right) \cos \left(\frac{\pi}{4} r_{5}\right)\right) \\
& -C_{6} \alpha_{1}^{3} \sin \left(\frac{\pi}{4} \alpha_{1}\right)+C_{7} \alpha_{1}^{3} \cos \left(\frac{\pi}{4} \alpha_{1}\right)-C_{8} \alpha_{2}^{3} \operatorname{sh}\left(\frac{\pi}{4} \alpha_{2}\right)-C_{9} \alpha_{2}^{3} \operatorname{ch}\left(\frac{\pi}{4} \alpha_{2}\right)=0 \\
& C_{1} r_{1}^{4} e^{\frac{\pi}{4} r_{1}}+C_{2} e^{\frac{\pi}{4}} r_{2}\left(\left(4 r_{2} r_{3}^{3}-4 r_{2}^{3} r_{3}\right) \sin \left(\frac{\pi}{4} r_{3}\right)+\left(r_{2}^{4}-6 r_{2}^{2} r_{3}^{2}+r_{3}^{4}\right) \cos \left(\frac{\pi}{4} r_{3}\right)\right) \\
& +C_{3} e^{\frac{\pi}{4}} r_{2}\left(\left(r_{2}^{4}-6 r_{2}^{2} r_{3}^{2}+r_{3}^{4}\right) \sin \left(\frac{\pi}{4} r_{3}\right)+\left(4 r_{2}^{3} r_{3}-4 r_{2} r_{3}^{3}\right) \cos \left(\frac{\pi}{4} r_{3}\right)\right) \\
& +C_{4} e^{\frac{\pi}{4} r_{4}}\left(\left(4 r_{4} r_{5}^{3}-4 r_{4}^{3} r_{5}\right) \sin \left(\frac{\pi}{4} r_{5}\right)+\left(r_{4}^{4}-6 r_{4}^{2} r_{5}^{2}+r_{5}^{4}\right) \cos \left(\frac{\pi}{4} r_{5}\right)\right) \\
& +C_{5} e^{\frac{\pi}{4} r_{4}}\left(\left(r_{4}^{4}-6 r_{4}^{2} r_{5}^{2}+r_{5}^{4}\right) \sin \left(\frac{\pi}{4} r_{5}\right)+\left(4 r_{4}^{3} r_{5}-4 r_{4} r_{5}^{3}\right) \cos \left(\frac{\pi}{4} r_{5}\right)\right) \\
& -C_{6} \alpha_{1}^{4} \cos \left(\frac{\pi}{4} \alpha_{1}\right)-C_{7} \alpha_{1}^{4} \sin \left(\frac{\pi}{4} \alpha_{1}\right)-C_{8} \alpha_{2}^{4} \operatorname{sh}\left(\frac{\pi}{4} \alpha_{2}\right)-C_{9} \alpha_{2}^{4} \operatorname{ch}\left(\frac{\pi}{4} \alpha_{2}\right)=0
\end{aligned}
$$


where

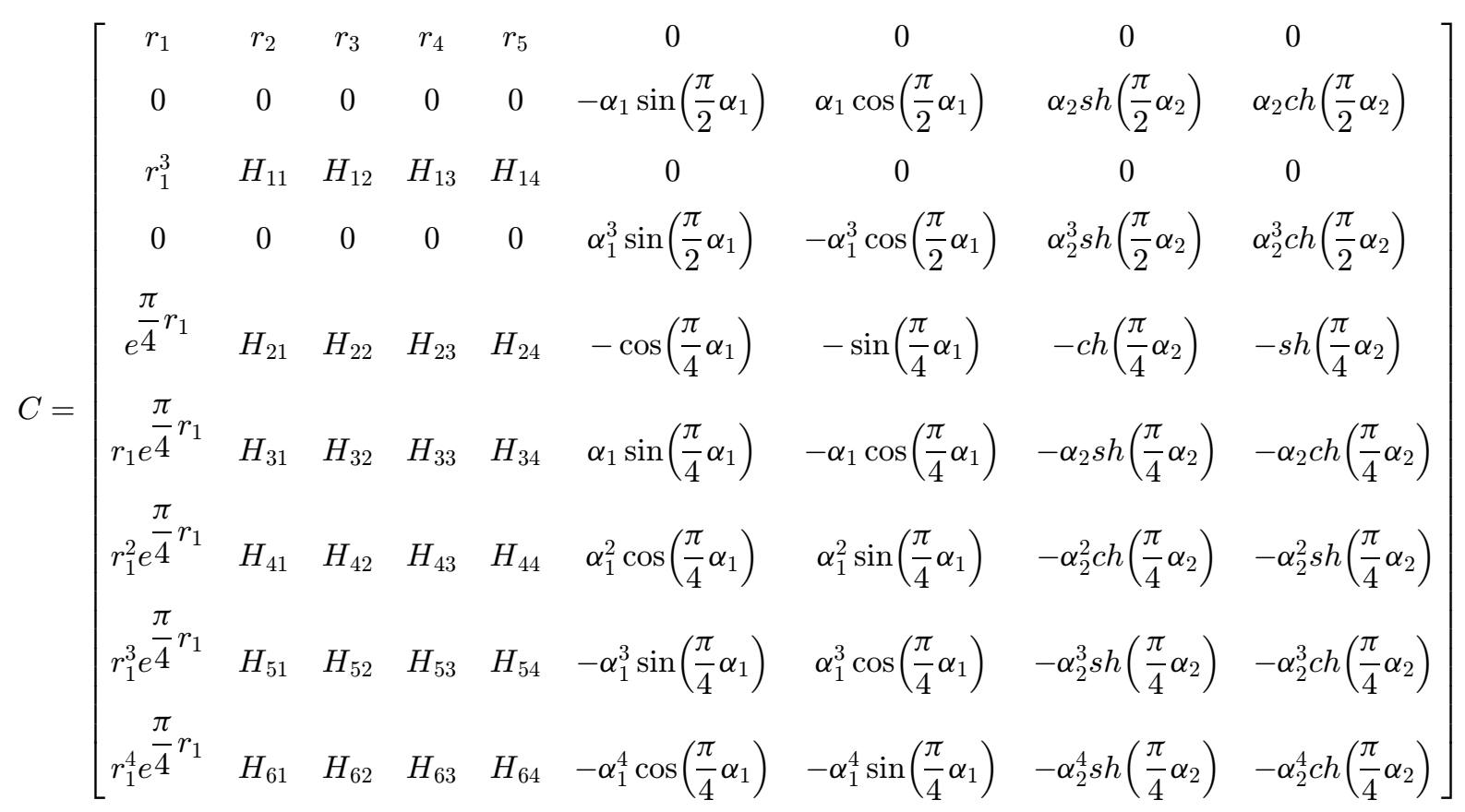

here

$$
\begin{gathered}
H_{61}=e^{\frac{\pi}{4} r_{2}}\left(\left(4 r_{2} r_{3}^{3}-4 r_{2}^{3} r_{3}\right) \sin \left(\frac{\pi}{4} r_{3}\right)+\left(r_{2}^{4}-6 r_{2}^{2} r_{3}^{2}+r_{3}^{4}\right) \cos \left(\frac{\pi}{4} r_{3}\right)\right), \\
H_{62}=e^{\frac{\pi}{4} r_{2}}\left(\left(r_{2}^{4}-6 r_{2}^{2} r_{3}^{2}+r_{3}^{4}\right) \sin \left(\frac{\pi}{4} r_{3}\right)+\left(4 r_{2}^{3} r_{3}-4 r_{2} r_{3}^{3}\right) \cos \left(\frac{\pi}{4} r_{3}\right)\right), \\
H_{63}=e^{\frac{\pi}{4} r_{4}}\left(\left(4 r_{4} r_{5}^{3}-4 r_{4}^{3} r_{5}\right) \sin \left(\frac{\pi}{4} r_{5}\right)+\left(r_{4}^{4}-6 r_{4}^{2} r_{5}^{2}+r_{5}^{4}\right) \cos \left(\frac{\pi}{4} r_{5}\right)\right), \\
H_{64}=e^{\frac{\pi}{4} r_{4}}\left(\left(r_{4}^{4}-6 r_{4}^{2} r_{5}^{2}+r_{5}^{4}\right) \sin \left(\frac{\pi}{4} r_{5}\right)+\left(4 r_{4}^{3} r_{5}-4 r_{4} r_{5}^{3}\right) \cos \left(\frac{\pi}{4} r_{5}\right)\right), \\
H_{51}=e^{\frac{\pi}{4} r_{2}}\left(\left(r_{3}^{3}-3 r_{2}^{2} r_{3}\right) \sin \left(\frac{\pi}{4} r_{3}\right)+\left(r_{2}^{3}-3 r_{2} r_{3}^{2}\right) \cos \left(\frac{\pi}{4} r_{3}\right)\right), \\
H_{52}=e^{\frac{\pi}{4} r_{2}}\left(\left(r_{2}^{3}-3 r_{2} r_{3}^{2}\right) \sin \left(\frac{\pi}{4} r_{3}\right)+\left(3 r_{2}^{2} r_{3}-r_{3}^{3}\right) \cos \left(\frac{\pi}{4} r_{3}\right)\right), \\
H_{53}=e^{\frac{\pi}{4} r_{4}}\left(\left(r_{5}^{3}-3 r_{4}^{2} r_{5}\right) \sin \left(\frac{\pi}{4} r_{5}\right)+\left(r_{4}^{3}-3 r_{4} r_{5}^{2}\right) \cos \left(\frac{\pi}{4} r_{5}\right)\right), \\
H_{54}=e^{\frac{\pi}{4} r_{4}}\left(\left(r_{4}^{3}-3 r_{4} r_{5}^{2}\right) \sin \left(\frac{\pi}{4} r_{5}\right)+\left(3 r_{4}^{2} r_{5}-r_{5}^{3}\right) \cos \left(\frac{\pi}{4} r_{5}\right)\right), \\
H_{41}=e^{\frac{\pi}{4} r_{2}}\left(\left(r_{2}^{2}-r_{3}^{2}\right) \cos \left(\frac{\pi}{4} r_{3}\right)-2 r_{2} r_{3} \sin \left(\frac{\pi}{4} r_{3}\right)\right), \\
H^{\frac{\pi}{4} r_{2}}\left(\left(r_{2}^{2}-r_{3}^{2}\right) \sin \left(\frac{\pi}{4} r_{3}\right)+2 r_{2} r_{3} \cos \left(\frac{\pi}{4} r_{3}\right)\right),
\end{gathered}
$$




$$
\begin{aligned}
& H_{43}=e^{\frac{\pi}{4} r_{4}}\left(\left(r_{4}^{2}-r_{5}^{2}\right) \cos \left(\frac{\pi}{4} r_{5}\right)-2 r_{4} r_{5} \sin \left(\frac{\pi}{4} r_{5}\right)\right) \\
& H_{44}=e^{\frac{\pi}{4} r_{4}}\left(\left(r_{4}^{2}-r_{5}^{2}\right) \sin \left(\frac{\pi}{4} r_{5}\right)+2 r_{4} r_{5} \cos \left(\frac{\pi}{4} r_{5}\right)\right), \\
& H_{31}=e^{\frac{\pi}{4} r_{2}}\left(r_{2} \cos \left(\frac{\pi}{4} r_{3}\right)-r_{3} \sin \left(\frac{\pi}{4} r_{3}\right)\right), \\
& H_{32}=e^{\frac{\pi}{4} r_{2}}\left(r_{2} \sin \left(\frac{\pi}{4} r_{3}\right)+r_{3} \cos \left(\frac{\pi}{4} r_{3}\right)\right), \\
& H_{33}=e^{\frac{\pi}{4} r_{4}}\left(r_{4} \cos \left(\frac{\pi}{4} r_{5}\right)-r_{5} \sin \left(\frac{\pi}{4} r_{5}\right)\right), \\
& H_{34}=e^{\frac{\pi}{4} r_{4}}\left(r_{4} \sin \left(\frac{\pi}{4} r_{5}\right)+r_{5} \cos \left(\frac{\pi}{4} r_{5}\right)\right), \\
& H_{21}=e^{\frac{\pi}{4} r_{2}} \cos \left(\frac{\pi}{4} r_{3}\right), H_{22}=e^{\frac{\pi}{4} r_{2}} \sin \left(\frac{\pi}{4} r_{3}\right), H_{23}=e^{\frac{\pi}{4} r_{4}} \cos \left(\frac{\pi}{4} r_{5}\right), \\
& H_{24}=e^{\frac{\pi}{4} r_{4}} \sin \left(\frac{\pi}{4} r_{5}\right), \\
& H_{11}=r_{2}^{3}-3 r_{2} r_{3}^{2}, H_{12}=3 r_{2}^{2} r_{3}-r_{3}^{3}, H_{13}=r_{4}^{3}-3 r_{4} r_{5}^{2}, H_{14}=3 r_{4}^{2} r_{5}-r_{5}^{3} \\
& X_{1}=\left[\begin{array}{lllllllll}
C_{1} & C_{2} & C_{3} & C_{4} & C_{5} & C_{6} & C_{7} & C_{8} & C_{9}
\end{array}\right]^{T}, \\
& D_{1}=\left[\begin{array}{lllllllll}
0 & 0 & 0 & 0 & 0 & 0 & 0 & 0 & 0
\end{array}\right]^{T}
\end{aligned}
$$

If there are non-zero solutions for equation (68), the frequencies of the flexible ring can be determined. determinant is zero, that is

Substituting the natural frequencies into following equation

See equation (69) below.

$$
\omega_{p}=\omega \sqrt{1-\zeta^{2}}
$$

Using equation (68), the constants $C_{j}$ of mode functions can be determined. Using equation (69), natural

where $\zeta=\frac{\eta l^{3}}{2 \omega\left(t_{0}-u_{0}\right)^{3} \rho A_{s}}$.

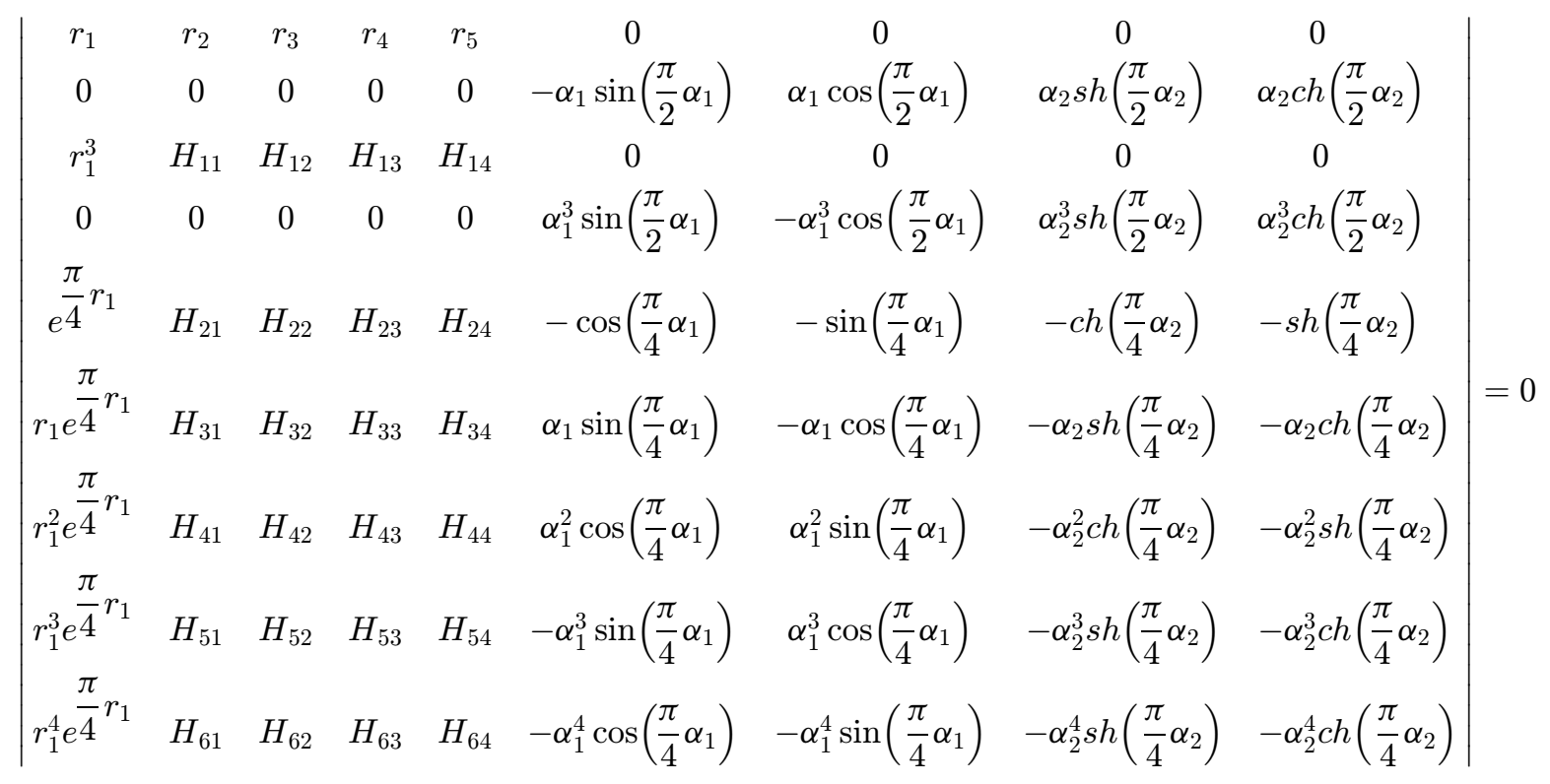


Table 1. Parameters of the system.

\begin{tabular}{llll}
\hline Parameters & Values & Parameters & Values \\
\hline$N$ & 200 & $R_{1}(\mathrm{~mm})$ & 10 \\
$x_{\mathrm{B}}(\mathrm{mm})$ & 10 & $t_{0}(\mu \mathrm{m})$ & 0.5 \\
$r(\mathrm{~mm})$ & 1.4 & $l(\mathrm{~mm})$ & 0.3 \\
$d(\mu \mathrm{m})$ & 50 & $E(\mathrm{MPa})$ & 95 \\
\hline
\end{tabular}

Table 2. Changes of natural frequency with $t_{0}(\mathrm{rad} / \mathrm{s})$.

\begin{tabular}{|c|c|c|c|c|c|c|c|}
\hline $\mathrm{t}_{0} / \mu \mathrm{m}$ & & 0.4 & 0.5 & 0.7 & 0.8 & 1 & 2 \\
\hline \multirow{4}{*}{ Order one } & $\omega_{e}$ & 6986.3 & 6986.3 & 6986.3 & 6986.3 & 6986.3 & 6986.3 \\
\hline & $\omega_{3}$ & 2328.7 & 4206.9 & 5688.3 & 6081.9 & 6554 & 6956.6 \\
\hline & $\omega_{e}-\omega_{3}$ & 4657.6 & 2279.4 & 1298 & 904.4 & 432.3 & 29.7 \\
\hline & $\omega_{e}$ & 34931.3 & 34931.3 & 34931.3 & 34931.3 & 34931.3 & 34931.3 \\
\hline \multirow[t]{3}{*}{ Order two } & $\omega_{3}$ & 31041.3 & 33379.2 & 34532.5 & 34698 & 34835.9 & 34925.3 \\
\hline & $\omega_{e}-\omega_{3}$ & 3890 & 1552.1 & 398.8 & 233.3 & 95.4 & 6 \\
\hline & $\omega_{e}$ & 81506.3 & 81506.3 & 81506.3 & 81506.3 & 81506.3 & 81506.3 \\
\hline \multirow[t]{3}{*}{ Order three } & $\omega_{3}$ & 79937.4 & 80857.2 & 81336.5 & 81406.7 & 81465.5 & 81503.8 \\
\hline & $\omega_{e}-\omega_{3}$ & 1568.9 & 649.1 & 169.8 & 99.6 & 40.8 & 2.5 \\
\hline & $\omega_{e}$ & 146711.4 & 146711.4 & 146711.4 & 146711.4 & 146711.4 & 146711.4 \\
\hline \multirow{2}{*}{ Order four } & $\omega_{3}$ & 145823.2 & 146348 & 146616.9 & 146656 & 146688.7 & 146710 \\
\hline & $\omega_{e}-\omega_{3}$ & 888.2 & 363.4 & 94.5 & 55.4 & 22.7 & 1.4 \\
\hline
\end{tabular}

Table 3. Changes of natural frequency with $r(\mathrm{rad} / \mathrm{s})$.

\begin{tabular}{|c|c|c|c|c|c|c|}
\hline$r / \mathrm{mm}$ & & 0.8 & 1 & 1.2 & 1.4 & 1.7 \\
\hline \multirow{4}{*}{ Order one } & $\omega_{e}$ & 21395.4 & 13693.1 & 9509.1 & 6986.3 & 4738.1 \\
\hline & $\omega_{3}$ & 19300.8 & 11149 & 6854.8 & 4206.9 & 949.9 \\
\hline & $\omega_{e}-\omega_{3}$ & 2094.6 & 2544.1 & 2654.3 & 2279.4 & 3788.2 \\
\hline & $\omega_{e}$ & 106977.1 & 68465.3 & 47545.4 & 34931.3 & 23690.4 \\
\hline \multirow[t]{3}{*}{ Order two } & $\omega_{3}$ & 106478.2 & 67683.8 & 46414.4 & 33379.2 & 21352.4 \\
\hline & $\omega_{e}-\omega_{3}$ & 498.9 & 781.5 & 1131 & 1552.1 & 2338 \\
\hline & $\omega_{e}$ & 249613.1 & 159752.4 & 110939.2 & 81506.3 & 55277.6 \\
\hline \multirow[t]{3}{*}{ Order three } & $\omega_{3}$ & 249399.9 & 159419.5 & 110460.8 & 80857.2 & 54328.3 \\
\hline & $\omega_{e}-\omega_{3}$ & 213.2 & 332.9 & 478.4 & 649.1 & 949.3 \\
\hline & $\omega_{e}$ & 449303.7 & 287554.3 & 199690.5 & 146711.4 & 99499.8 \\
\hline \multirow{2}{*}{ Order four } & $\omega_{3}$ & 449185.1 & 287369.1 & 199423.6 & 146348 & 98963.4 \\
\hline & $\omega_{e}-\omega_{3}$ & 118.6 & 185.2 & 266.9 & 363.4 & 536.4 \\
\hline
\end{tabular}

From equation (70), the effects of the air damping on the natural frequencies can be given.

\section{Results and discussion}

Using equations given in this paper, the free vibration of the micro electromagnetic harmonic drive system is investigated. Parameters of the system are shown in Table 1.
Table 1 shows that the clearance between the flexible ring and stator is $500 \mathrm{~nm}$ and the effects of the Van der Walls force on the natural frequencies are quite significant and should be considered. Tables $2-4$ give the first four orders of the natural frequencies and their changes along with system parameters. Results show:

- Considering molecule force, the natural frequencies of the flexible ring are decreased. With decreasing clearance 
Table 4. Changes of natural frequency with $d(\mathrm{rad} / \mathrm{s})$.

\begin{tabular}{|c|c|c|c|c|c|c|c|}
\hline$d / \mu \mathrm{m}$ & & 30 & 40 & 50 & 80 & 100 & 150 \\
\hline \multirow{4}{*}{ Order one } & $\omega_{e}$ & 4191.8 & 5589 & 6986.3 & 11178 & 13972.5 & 20958.8 \\
\hline & $\omega_{3}$ & 954.1 & 1863 & 4206.9 & 9182.3 & 12418.9 & 20157.8 \\
\hline & $\omega_{e}-\omega_{3}$ & 3237.7 & 3726 & 2279.4 & 1995.7 & 1553.6 & 801 \\
\hline & $\omega_{e}$ & 20958.8 & 27945 & 34931.3 & 55890.1 & 69862.6 & 104793.9 \\
\hline \multirow[t]{3}{*}{ Order two } & $\omega_{3}$ & 16370.1 & 25477.4 & 33379.2 & 55291.8 & 69480.5 & 104624.3 \\
\hline & $\omega_{e}-\omega_{3}$ & 4588.7 & 2467.6 & 1552.1 & 598.3 & 382.1 & 169.6 \\
\hline & $\omega_{e}$ & 48903.8 & 65205.1 & 81506.3 & 130410.1 & 163012.7 & 244519 \\
\hline \multirow[t]{3}{*}{ Order three } & $\omega_{3}$ & 47146.6 & 64197.5 & 80857.2 & 130155.2 & 162849.4 & 244446.4 \\
\hline & $\omega_{e}-\omega_{3}$ & 1757.2 & 1007.6 & 649.1 & 254.9 & 163.3 & 72.6 \\
\hline & $\omega_{e}$ & 88026.8 & 117369.1 & 146711.4 & 234738.2 & 293422.8 & 440134.2 \\
\hline \multirow[t]{2}{*}{ Order four } & $\omega_{3}$ & 87014.4 & 116800.9 & 146348 & 234596.4 & 293332 & 440093.9 \\
\hline & $\omega_{e}-\omega_{3}$ & 1012.4 & 568.2 & 363.4 & 141.8 & 90.8 & 40.3 \\
\hline
\end{tabular}

between the flexible ring and stator, the natural frequencies of the flexible ring are decreased more rapidly. This is due to the increased effects of electromagnetic force and molecular force on the flexible ring, resulting in the stiffness reduction of the coupling system.

- As clearance between the flexible ring and stator is relatively large $\left(t_{0}>1 \mu \mathrm{m}\right)$, the decrease of the natural frequencies of the flexible ring is not obvious. At $t_{0}=1 \mu \mathrm{m}$, the relative error between the first order of the natural frequencies with and without considering molecule force is equal to $\left(\omega_{\mathrm{e}}-\omega_{3}\right) / \omega_{\mathrm{e}}=6.2 \%$. At $t_{0}=2 \mu \mathrm{m}$, the relative error is: $\left(\omega_{\mathrm{e}}-\omega_{3}\right) / \omega_{\mathrm{e}}=0.4 \%$. At $t_{0}=0.8 \mu \mathrm{m}$, the relative error between natural frequencies with and without considering molecule force is equal to: $\left(\omega_{\mathrm{e}}-\omega_{3}\right) / \omega_{\mathrm{e}}=12.9 \%$. At $t_{0}=0.5 \mu \mathrm{m}$ and $t_{0}=0.4 \mu \mathrm{m}$, the relative error between natural frequencies is equal to: $\left(\omega_{e}-\omega_{3}\right) / \omega_{\mathrm{e}}=32.6 \%$. $\left(t_{0}=0.5 \mu \mathrm{m}\right)$ and $t_{0}=0.4 \mu \mathrm{m}$, and $\left(\omega_{e}-\omega_{3}\right) / \omega_{\mathrm{e}}=66.7 \%$. $\left(t_{0}=0.4 \mu \mathrm{m}\right)$.

- As the order number of the vibration modes increases, effects of the molecule force on the natural frequencies of the flexible ring becomes weak as well. For example, at $t_{0}=0.4 \mu \mathrm{m}$, the relative error between the second order of the natural frequencies with and without considering molecule force is reduced to be $11.1 \%$; the relative error between the third order of the natural frequencies is reduced to be $1.9 \%$; and the relative error between the fourth order of the natural frequencies is reduced to be $0.6 \%$.

- With increasing radius of the flexible ring, its natural frequencies are decreased. For a relatively large radius of the flexible ring, the relative error between the natural frequencies with and without considering molecule force becomes large. It shows that effects of the molecule force on the natural frequencies of the flexible ring increases with increasing radius of the flexible ring. As the order number of the vibration modes increases, effects of the molecule force on relationship between the natural frequencies and radius of the flexible ring becomes weak.
At radius $r=1.4 \mathrm{~mm}$ decrease of the natural frequencies caused by molecule force is $6.2 \%$ for mode one, $0.3 \%$ for mode two, $0.05 \%$ for mode three, and $0.02 \%$ for mode 4 .

- With increasing thickness of the flexible ring, the natural frequencies of the flexible ring are increased. For a relatively large thickness of the flexible ring, the relative error between the natural frequencies with and without considering molecule force becomes small. It shows that effects of the molecule force on the natural frequencies of the flexible ring decreases with increasing thickness of the flexible ring. As the order number of the vibration modes increases, effects of the molecule force on relationship between the natural frequencies and thickness of the flexible ring becomes weak. At thickness $d=40 \mathrm{~mm}$, decrease of the natural frequencies caused by molecule force is $66.7 \%$ for mode one, $8.8 \%$ for mode two, $1.55 \%$ for mode three, and $0.48 \%$ for mode 4 .

In a word, in dynamics performance design of the drive system, to determine its natural frequency accurately, the effects of the molecule force on the natural frequencies should be considered for smaller clearance between the flexible ring and stator, smaller thickness of the flexible ring and larger radius of the flexible ring.

Substituting above natural frequencies into equations (32), (36) and (40), the first four orders of the vibration modes can be obtained (see Fig. 3). It shows:

- As the molecule force is considered, for mode 1 , at $\theta=0$ and $\theta=\pi$, the amplitudes of the flexible ring vibrations decrease; at $\theta=\pi / 2$ and $\theta=3 \pi / 2$, the amplitudes of the flexible ring vibrations increase obviously. It is because there is larger molecule force near $\theta=0$ and $\theta=\pi$ than other places.

- As the order number of the vibration modes increases, effects of the molecule force on the vibration modes of the flexible ring becomes weak as well. For mode 1, difference between modes with and without molecule force is 0.8376; for mode 2, 3 and 4, the difference between modes are $0.0464,0.0168$, and 0.0025 , respectively. 


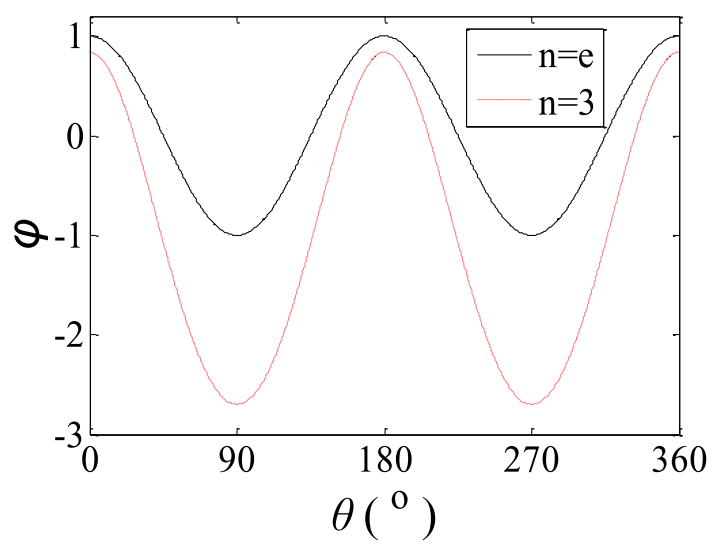

(a)

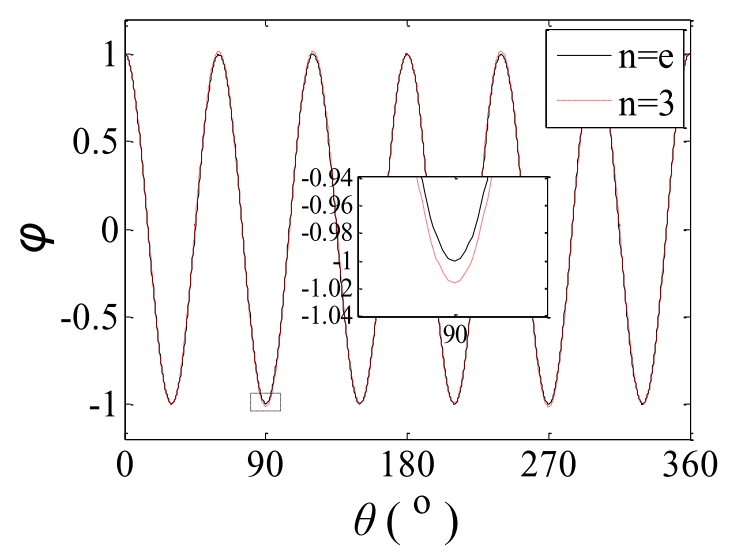

(c)

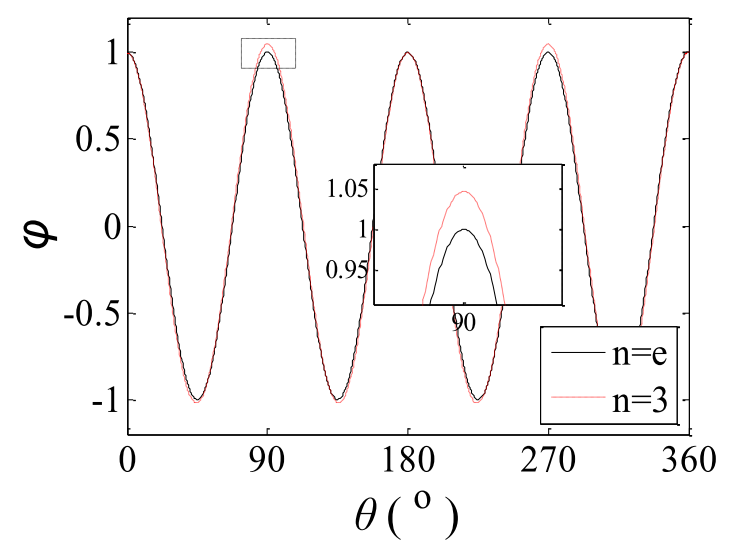

(b)

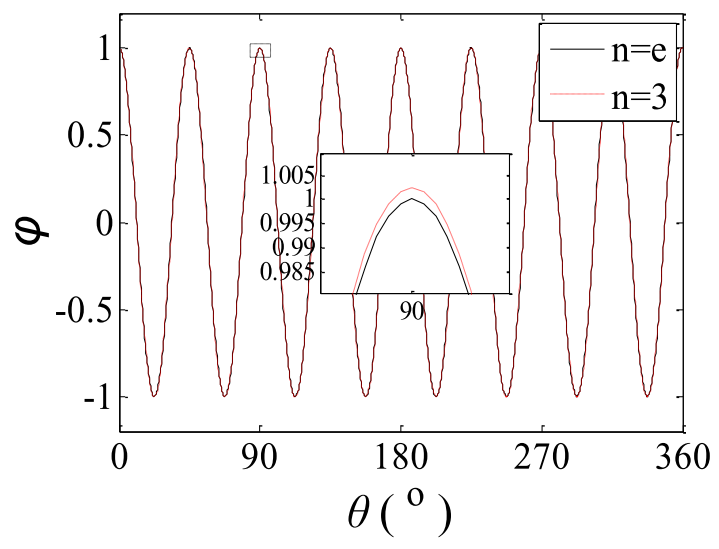

(d)

Fig. 3. Differences between modes with and without molecule force. (a) Mode 1 (b) mode 2 (c) mode 3 (d) mode 4.

From mode analysis, it can be known that effects of the molecule force on the vibration modes are obvious only for modes 1 and 2. Here, for modes 1 and 2, effects of the molecule force on relationship between the vibration modes and other parameters are investigated (see Figs. 4-6). Results show:

- As the clearance between the flexible ring and stator is relatively small, effects of the molecule force on vibration amplitudes of the flexible ring become more obvious. At the clearance $t_{0}=0.4 \mu \mathrm{m}$, the difference between the maximum vibration amplitudes for mode one with and without considering molecule force is 2.287. At the clearance $t_{0}=0.5 \mu \mathrm{m}, t_{0}=1 \mu \mathrm{m}$ and $t_{0}=2 \mu \mathrm{m}$, the difference between the maximum vibration amplitudes is $1.700,0.164$ and 0.008 , respectively. For mode two, the difference between the maximum vibration amplitudes is 0.136 for $t_{0}=0.4,0.047$ for, 0.0002 for $t_{0}=2 \mu \mathrm{m}$.

- As the radius $r$ of the flexible ring increases (from 0.8 to $1.7 \mathrm{~mm})$, vibration amplitudes of the flexible ring increase as well. Near $\theta=\pi / 2$ and $\theta=3 \pi / 2$, vibration amplitudes of the flexible ring increase are increased more obviously. Near $\theta=\pi / 2$ and $\theta=3 \pi / 2$, the difference between the maximum vibration amplitudes with and without considering molecule force is large as well. For mode 1 , the difference between the maximum vibration amplitudes with and without considering molecule force is 0.2861 at $r=0.8 \mathrm{~mm}, 0.69$ at $r=1 \mathrm{~mm}$, and 2.045 at $r=1.7 \mathrm{~mm}$. For mode 2, the difference between the maximum vibration amplitudes with and without considering molecule force is 0.005 at $r=0.8 \mathrm{~mm}$, 0.012 at $r=1 \mathrm{~mm}$, and 0.112 at $r=1.7 \mathrm{~mm}$.

The results show that effects of the molecule force on the maximum vibration amplitudes increases with increasing the radius $r$ of the flexible ring.

- As the thickness $d$ of the flexible ring increases (from $30 \mu \mathrm{m}$ to $150 \mu \mathrm{m}$ ), vibration amplitudes of the flexible ring decrease. As the thickness $d$ of the flexible ring increases, effects of the molecule force on vibration amplitudes of the flexible ring become weak. For mode 1, the difference between the maximum vibration amplitudes with and without considering molecule force is 2.509 at $d=30 \mu \mathrm{m}, 1.7$ at $d=50 \mu \mathrm{m}$, and 0.09547 at $d=150 \mu \mathrm{m}$. For mode 2 , the difference between the maximum vibration amplitudes with and without considering molecule force is 0.3066 at $d=30 \mu \mathrm{m}$, 0.04698 at $d=150 \mu \mathrm{m}$, and 0.0056 at $d=150 \mu \mathrm{m}$. 


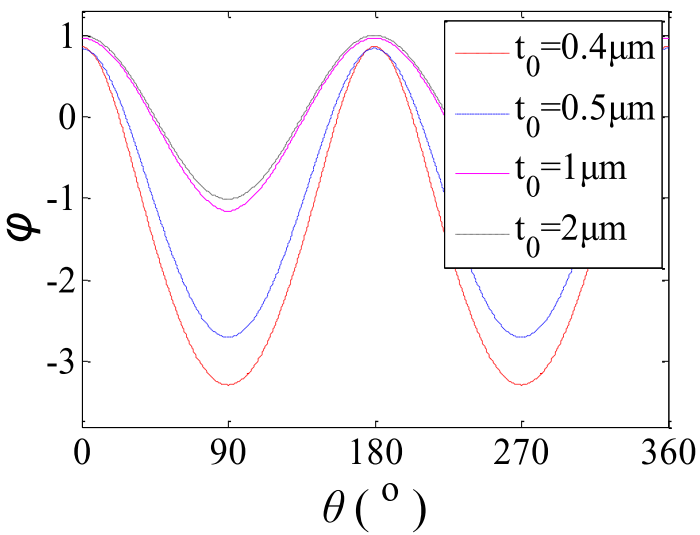

(a)

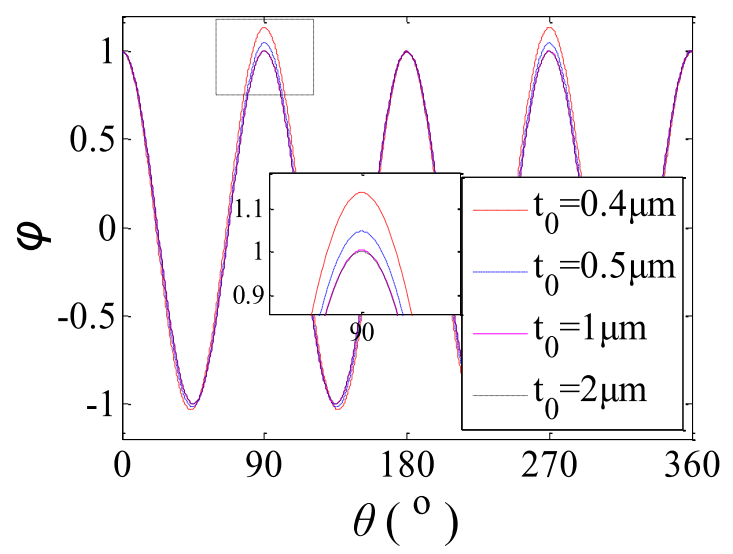

(c)

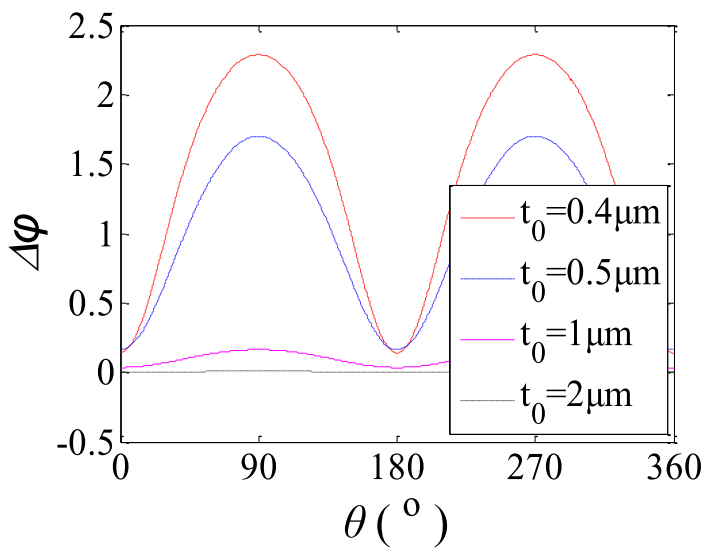

(b)

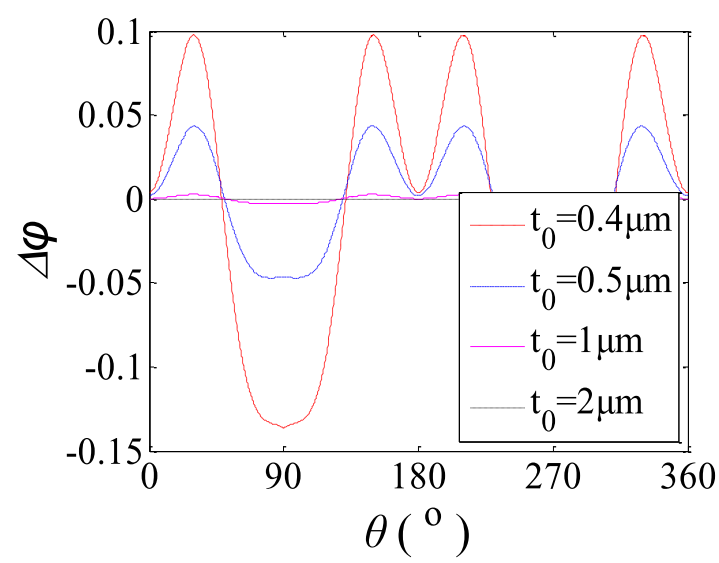

(d)

Fig. 4. Vibration modes for different clearance $t_{0}$. (a) Mode 1 (b) $\Delta \phi=\phi_{\mathrm{e}}-\phi_{3}$ for mode 1 (c) mode 2 (d) $\Delta \phi=\phi_{\mathrm{e}}-\phi_{3}$ for mode 2 .

It can be seen that effects of the molecule force on the maximum vibration amplitudes increases with decreasing the thickness $d$ of the flexible ring and order number of the modes.

Using equations (69) and (70), the effects of the friction force and air damping on the natural frequencies are investigated. The parameters of the drive system are shown in Table 1. The effects of the friction force are given in Tables 5-8. The effects of the damping are given in Tables 9-12. Results show:

- When considering friction force, the natural frequencies of the flexible ring are decreased. With increasing order number of the modes, the effects of the friction force on the natural frequencies reduce rapidly and the effects can be neglected when the order number of the modes is above 2 .

- When the clearance between the flexible ring and stator is decreased, the effects of the friction force on the natural frequencies become more significant. When the radius of the flexible ring is increased, the effects of the friction force on the natural frequencies decrease.
- When the thickness of the flexible ring increases, the frequency difference $\left(\omega_{3}-\omega_{\mathrm{t}}\right)$ increases. This shows that the effects of the friction force on the natural frequencies become more significant for large thickness of the flexible ring. When the current in the coils increases, the frequency difference $\left(\omega_{3}-\omega_{t}\right)$ decreases. This shows that the effects of the friction force on the natural frequencies reduce for large coil current.

- When considering air damping force, the natural frequencies of the flexible ring are also decreased. With increasing order number of the modes, the effects of the air damping force on the natural frequencies reduce as well.

- When the clearance between the flexible ring and stator is decreased, the frequency difference $\left(\omega_{\mathrm{t}}-\omega_{\mathrm{p}}\right)$ increases which shows that the effects of the air damping force on the natural frequencies increases with decreasing the clearance.

- When the radius of the flexible ring is increased, the frequency difference $\left(\omega_{3}-\omega_{\mathrm{t}}\right)$ increases which shows that the effects of the air damping force on the natural frequencies increases with decreasing the radius.

- When the thickness of the flexible ring increases, the frequency difference $\left(\omega_{3}-\omega_{t}\right)$ decreases which shows that 
Table 5. Changes of natural frequency with $t_{0}$ with and without friction force $(\mathrm{rad} / \mathrm{s})$.

\begin{tabular}{llcccccr}
\hline$t_{0} / \mu \mathrm{m}$ & & 0.3 & 0.4 & 0.5 & 0.6 & 0.8 & 1 \\
\hline \multirow{3}{*}{ Order 1} & $\omega_{3}$ & 5711.9 & 6634.5 & 6848.1 & 6921 & 6966 & 6978.1 \\
& $\omega_{t}$ & 2328.7 & 2454.9 & 2372.4 & 2348.6 & 2334.8 & 2331.2 \\
& $\omega_{3}-\omega_{t}$ & 3383.2 & 4179.6 & 4475.7 & 4572.4 & 4631.2 & 4646.9 \\
\multirow{3}{*}{ Order 2} & $\omega_{3}$ & 34544.2 & 34855.3 & 34902.9 & 34918.1 & 34927.2 & 34929.6 \\
& $\omega_{t}$ & 34544.2 & 34855.3 & 34902.9 & 34918.1 & 34927.2 & 34929.6 \\
& $\omega_{3}-\omega_{t}$ & 0 & 0 & 0 & 0 & 0 & 0 \\
\multirow{2}{*}{ Order 3 } & $\omega_{3}$ & 81341.5 & 81473.8 & 81494.2 & 81500.7 & 81504.6 & 81505.6 \\
& $\omega_{t}$ & 81341.5 & 81473.8 & 81494.2 & 81500.7 & 81504.6 & 81505.6 \\
& $\omega_{3}-\omega_{t}$ & 0 & 0 & 0 & 0 & 0 & 0 \\
\hline
\end{tabular}

Table 6. Changes of natural frequency with $r$ with and without friction force ( $\mathrm{rad} / \mathrm{s})$.

\begin{tabular}{llcccccr}
\hline$r / \mathrm{mm}$ & & 0.8 & 1 & 1.2 & 1.3 & 1.4 & 1.45 \\
\hline \multirow{4}{*}{ Order 1} & $\omega_{3}$ & 21069.1 & 13163.8 & 8693.7 & 7094.5 & 5711.9 & 4988.4 \\
& $\omega_{t}$ & 7233.2 & 4744.6 & 3522.8 & 3288.4 & 2328.7 & 2170.9 \\
& $\omega_{3}-\omega_{t}$ & 13835.9 & 8419.2 & 5170.9 & 3806.1 & 3383.2 & 2817.5 \\
& $\omega_{3}$ & 106910.4 & 68353.2 & 47356.4 & 40255.5 & 34544.2 & 32007.7 \\
Order 2 & $\omega_{t}$ & 106910.4 & 68353.2 & 47356.4 & 40255.5 & 34544.2 & 32007.7 \\
& $\omega_{3}-\omega_{t}$ & 0 & 0 & 0 & 0 & 0 & 0 \\
& $\omega_{3}$ & 249584.6 & 159704.4 & 110858.4 & 94418.5 & 81341.5 & 75745.5 \\
Order 2 & $\omega_{t}$ & 249584.6 & 159704.4 & 110858.4 & 94418.5 & 81341.5 & 75745.5 \\
& $\omega_{3}-\omega_{t}$ & 0 & 0 & 0 & 0 & 0 & 0 \\
\hline
\end{tabular}

Table 7. Changes of natural frequency with $d$ with and without friction force $(\mathrm{rad} / \mathrm{s})$.

\begin{tabular}{llccccrr}
\hline$d / \mu \mathrm{m}$ & & 48 & 50 & 60 & 80 & 100 & 150 \\
\hline \multirow{3}{*}{ Order 1} & $\omega_{3}$ & 5200.3 & 5711.9 & 7605.4 & 10774.3 & 13721.5 & 20849.6 \\
& $\omega_{t}$ & 2235.6 & 2328.7 & 3147.1 & 3862.0 & 4736.2 & 7019.0 \\
& $\omega_{3}-\omega_{t}$ & 2964.7 & 3383.2 & 4458.3 & 6912.3 & 8985.3 & 13830.6 \\
\multirow{3}{*}{ Order 2} & $\omega_{3}$ & 33002.9 & 34544.2 & 41734.4 & 55804.9 & 69811.1 & 104771.9 \\
& $\omega_{t}$ & 33002.9 & 34544.2 & 41734.4 & 55804.9 & 69811.1 & 104771.9 \\
& $\omega_{3}-\omega_{t}$ & 0 & 0 & 0 & 0 & 0 & 0 \\
\multirow{2}{*}{ Order 3 } & $\omega_{3}$ & 78020.4 & 81341.5 & 97729.3 & 130373.7 & 162990.6 & 244509.6 \\
& $\omega_{t}$ & 78020.4 & 81341.5 & 97729.3 & 130373.7 & 162990.6 & 244509.6 \\
& $\omega_{3}-\omega_{t}$ & 0 & 0 & 0 & 0 & 0 & 0 \\
\hline
\end{tabular}

Table 8. Changes of natural frequency with $I$ with and without friction force ( $\mathrm{rad} / \mathrm{s})$.

\begin{tabular}{llccccr}
\hline$I / \mathrm{A}$ & & 0.05 & 0.1 & 0.13 & 0.15 & 0.16 \\
\hline \multirow{3}{*}{ Order 1} & $\omega_{3}$ & 5850.7 & 5711.9 & 5546.1 & 5347 & 5125.5 \\
& $\omega_{t}$ & 2328.7 & 2328.7 & 2328.7 & 2328.7 & 2328.7 \\
& $\omega_{3}-\omega_{t}$ & 3522.0 & 3383.2 & 3217.4 & 3018.3 & 2796.8 \\
\multirow{3}{*}{ Order 2} & $\omega_{3}$ & 34608.2 & 34544.2 & 34456.1 & 34332.1 & 34171.1 \\
& $\omega_{t}$ & 34608.2 & 34544.2 & 34456.1 & 34332.1 & 34171.1 \\
& $\omega_{3}-\omega_{t}$ & 0 & 0 & 0 & 0 & 0 \\
\multirow{2}{*}{ Order 3 } & $\omega_{3}$ & 81368.6 & 81341.5 & 81304.2 & 81251.9 & 81184.4 \\
& $\omega_{t}$ & 81368.6 & 81341.5 & 81304.2 & 81251.9 & 81184.4 \\
& $\omega_{3}-\omega_{t}$ & 0 & 0 & 0 & 0 & 0 \\
\hline
\end{tabular}


Table 9. Changes of natural frequency with $t_{0}$ with and without damping $(\mathrm{rad} / \mathrm{s})$.

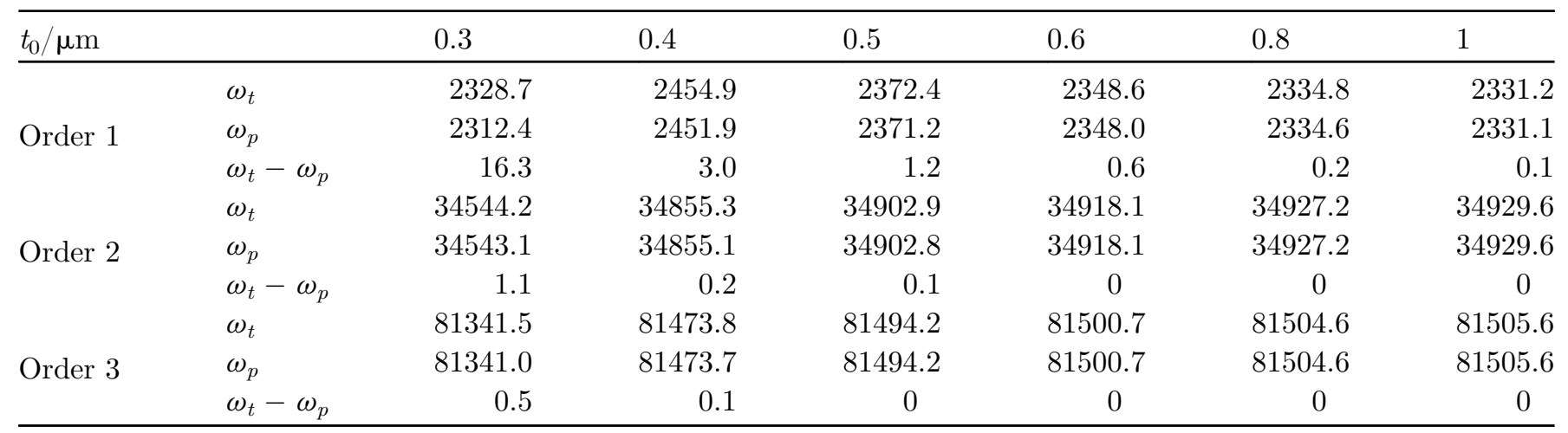

Table 10. Changes of natural frequency with $r$ with and without damping ( $\mathrm{rad} / \mathrm{s})$.

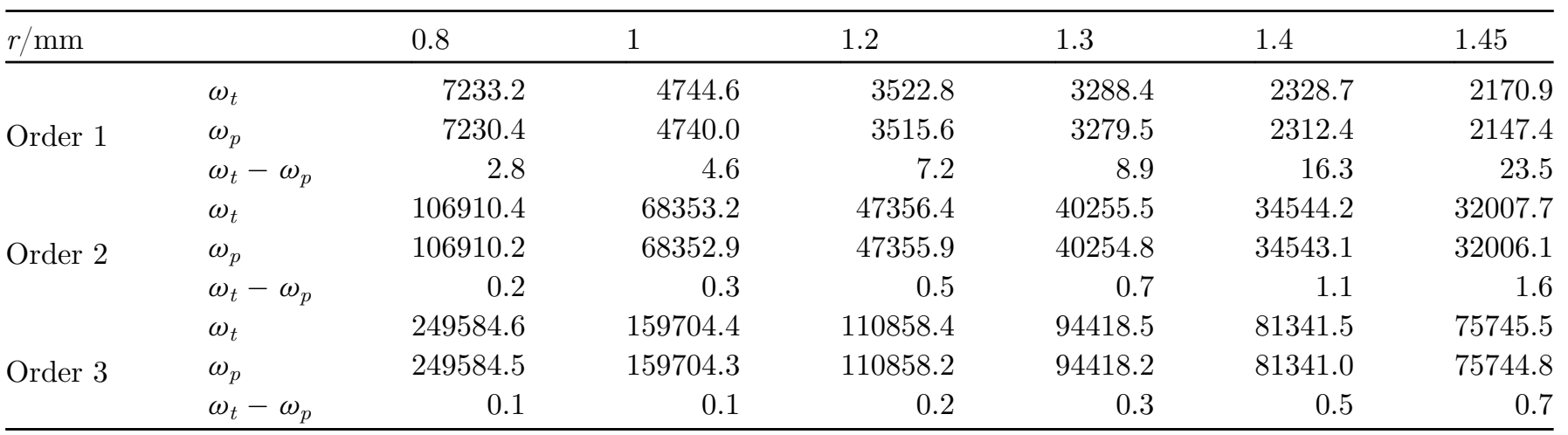

Table 11. Changes of natural frequency with $d$ with and without damping $(\mathrm{rad} / \mathrm{s})$.

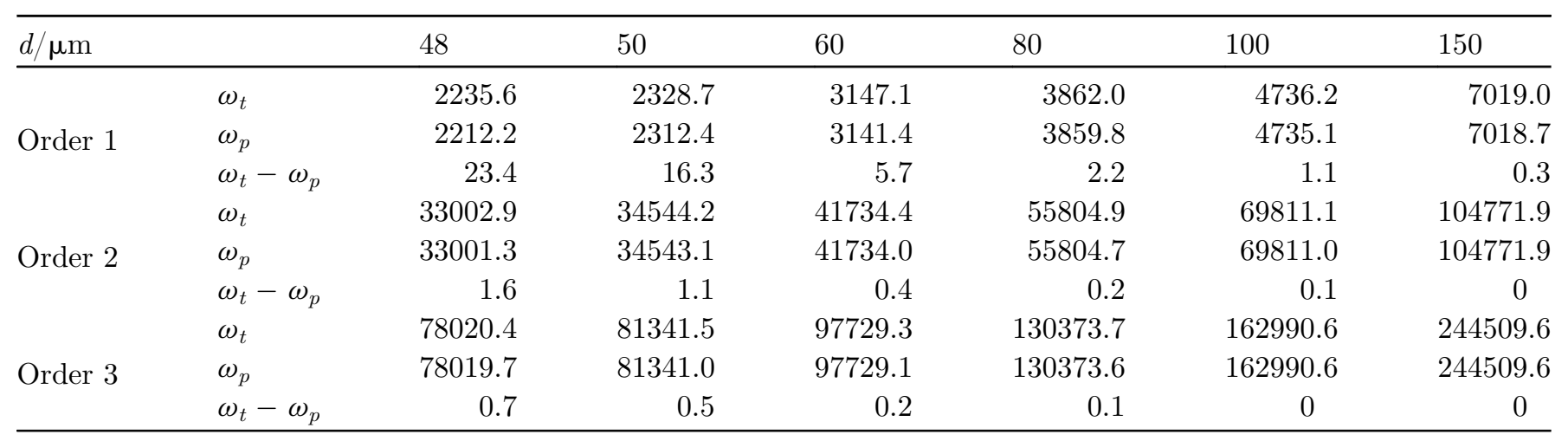

Table 12. Changes of natural frequency with $I$ with and without damping $(\mathrm{rad} / \mathrm{s})$.

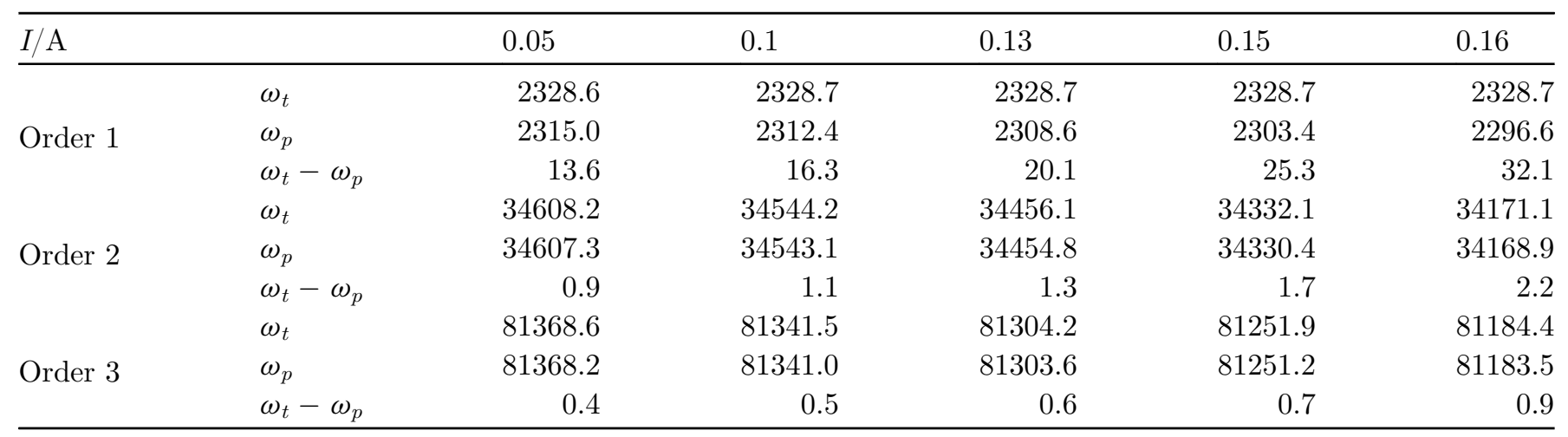




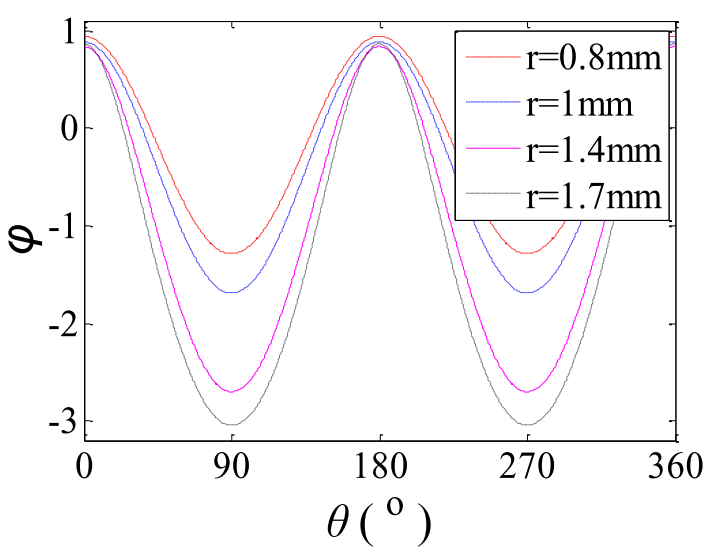

(a)

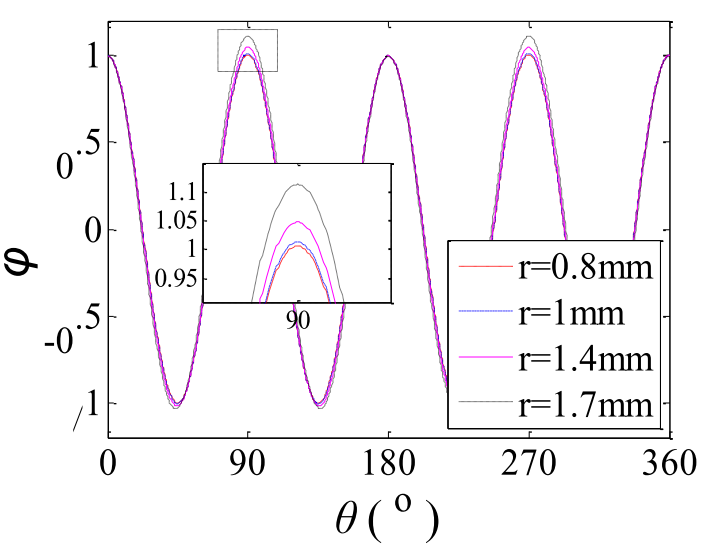

(c)

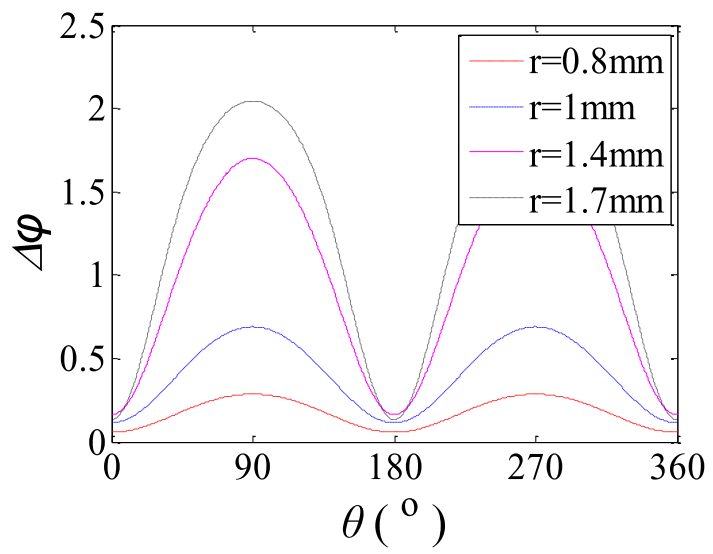

(b)

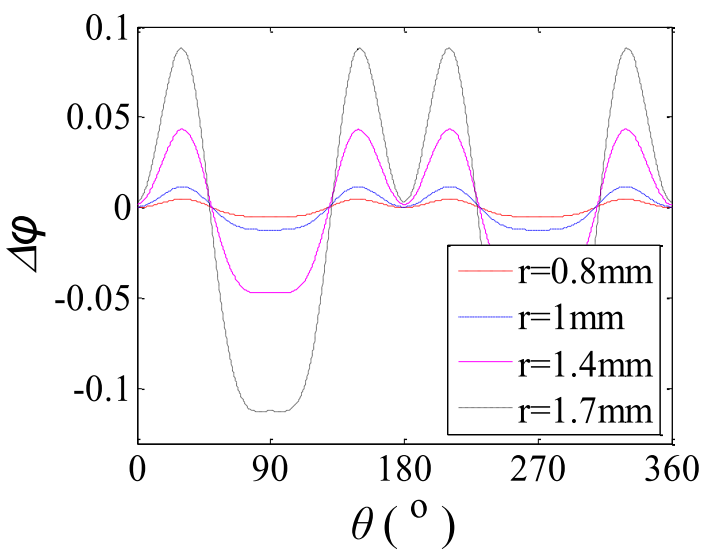

(d)

Fig. 5. Vibration modes for different radius $r$. (a) Mode 1 (b) $\Delta \phi=\phi_{\mathrm{e}}-\phi_{3}$ for mode 1 (c) mode 2 (d) $\Delta \phi=\phi_{\mathrm{e}}-\phi_{3}$ for mode 2 .

the effects of the air damping force on the natural frequencies become weak for large thickness of the flexible ring.

- When the current in the coils increases, the frequency difference $\left(\omega_{3}-\omega_{\mathrm{t}}\right)$ increases which shows that the effects of the air damping force on the natural frequencies increases for large coil current.

To illustrate the theoretical analysis, FEM software, ANSYS, is used to simulate the dynamics performance of the flexible ring. The simulating process is as follows:

- FEM model of the flexible ring is produced. The density, Young's modulus and Poisson's ratio of the flexible ring material are set.

- At $\theta=0$ and $\theta=\pi / 2$, the movement and rotation in other directions of the flexible ring are restricted, only the movement in radial direction is allowed.

- Calculated electromagnetic force and molecular force are converted into the force per unit area applied to the flexible ring. The electromagnetic force and molecular force are 0.0785 and $0.2156 \mathrm{~N} / \mathrm{m}^{2}$, respectively. The direction of the forces is radial to the outside of the flexible wheel.
Table 13. Comparison of calculated and simulated natural frequencies.

\begin{tabular}{|c|c|c|c|}
\hline & Calculated & Simulated $(\mathrm{Hz})$ & Error $(\%)$ \\
\hline 1 & 909.077 & 1095.1 & 16.99 \\
\hline 2 & 5497.88 & 5921.1 & 7.15 \\
\hline 3 & 12945.9 & 13977 & 7.38 \\
\hline 4 & 23335.23 & 25132 & 7.15 \\
\hline
\end{tabular}

Using the FEM model, the natural frequencies of the flexible wheel are obtained and compared with the calculated ones (see Tab. 13). Table 13 show:

The calculated and simulated natural frequencies are in good agreement with each other. The relative error between them is below $17 \%$. This illustrates theoretical analysis of the paper.

\section{Conclusions}

In this paper, considering Van der Waals force, dynamics equation of the flexible ring for the micro electromagnetic 


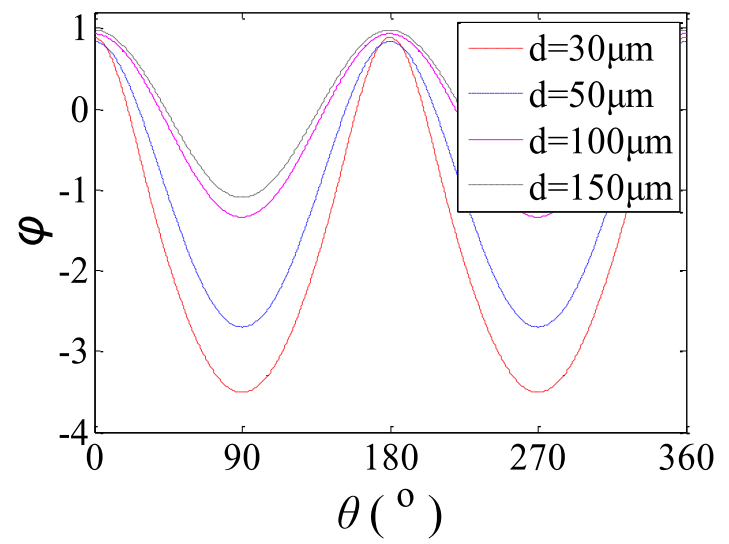

(a)

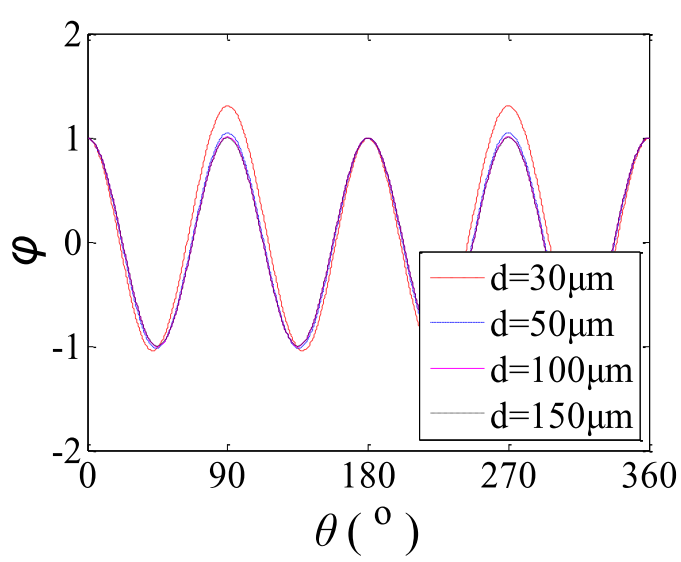

(c)

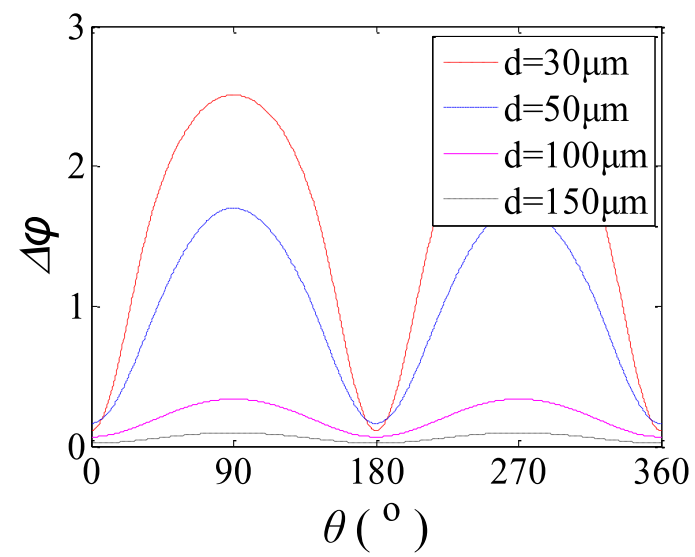

(b)

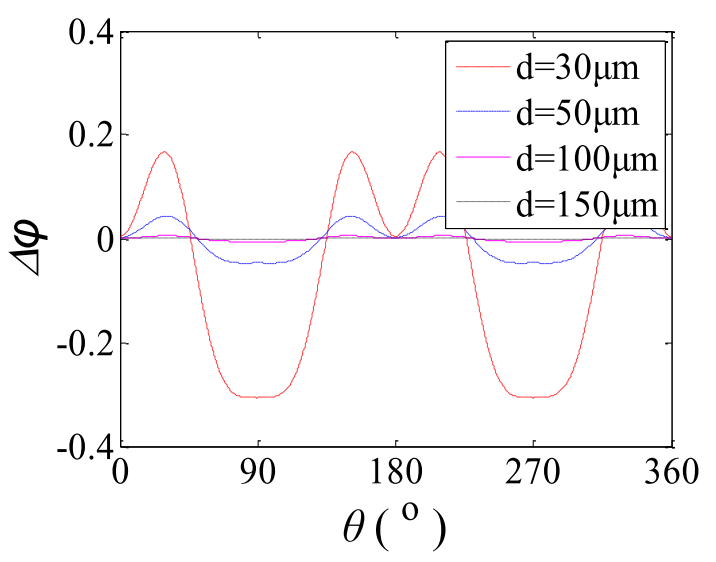

(d)

Fig. 6. Vibration modes for different thickness $d$. (a) Mode 1 (b) $\Delta \phi=\phi_{\mathrm{e}}-\phi_{3}$ for mode 1 (c) mode 2 (d) $\Delta \phi=\phi_{\mathrm{e}}-\phi_{3}$ for mode 2 .

harmonic drive system is proposed. Using the equations, effects of the molecule force on the natural frequencies and vibration modes of the drive system are investigated. Results show:

- Considering molecule force, natural frequencies of the flexible ring are reduced. For lower order modes, the effects of the molecule force on the natural frequencies are more obvious. For smaller clearance between the flexible ring and stator, smaller thickness of the flexible ring and larger radius of the flexible ring, the effects of the molecule force on the natural frequencies are more obvious.

- Considering molecule force, vibration modes of the flexible ring are changed. At some positions, the vibration amplitudes are decreased; at other positions, the vibration amplitudes are increased. For lower order modes, the effects of the molecule force on the vibration modes are more obvious. For smaller clearance between the flexible ring and stator, smaller thickness of the flexible ring and larger radius of the flexible ring, the effects of the molecule force on the vibration modes are more obvious.
- When considering friction force and air damping force, the natural frequencies of the flexible ring are decreased.

\section{References}

[1] C. Wang, P. Yang, L. Zhang, Summary of status on the harmonic gear driving technology, Journal of Mechanical Transmission 30, 536-567 (2006)

[2] K. Davey, A harmonic gear like motor. IEEE International Electric Machines and Drives Conference, Miami, FL, USA. IEEE, pp. 1795-1800, (2009)

[3] W. Trimmer, R. Jebens, Harmonic electrostatic motors, Sensors Actuators 20, 17-24 (1989)

[4] Y. Sun, Z. Gu, G. Liu, Q. Li, Design of measurement and control system for the efficiency test of spacecraft harmonic drive mechanism, 8th International Conference on Mechanical and Aerospace Engineering, pp. 704-708 (2017)

[5] R. Liu, Y. Zhang, Y. Wang, An efficient conformal mapping method for air gap magnetic field analytical calculation in an eccentric magnetic harmonic gear, Proceedings of 2015 IEEE 
International Conference on ID5059 Applied Superconductivity and Electromagnetic Devices Shanghai, China, November 20-23 (2015)

[6] D. Herdeg, Electromagnet harmonic drive low inertia servo actuator. AD44 2879, Dec 1963

[7] C. Janes, Electro-magnetic actuator. US Patent, No. 3200 668. Aug 1959

[8] O. Kayabasi, F. Erzincanli, Shape optimization of tooth profile of aflexspline for aharmonic drive by finite element modeling, Materials \& Design 28, 441-447 (2007)

[9] D. Nista, A. Viola, Electromagnetic harmonic motor with continuous control of position and torque. Proceedings of the 27th Applications in the Transportation Industries, Aachen, pp. 487-491 (1994)

[10] J. Rens, R. Clark, S. Calverley, Design, analysis and realization of anovel magnetic harmonic gear, Electrical Machines, ICEM 2008 (18th International Conference), pp. 1-4 (2008)

[11] J. Rens, K. Atallah, S. Calverley, A novel magnetic harmonic gear, Transactions on Industry Applications (IEEE) 46 , 206-212 (2010)

[12] T. Tjahjowidodo, F. Al-Bender, B. Van, Theoretical modelling and experimental identification of nonlinear torsional behaviour in harmonic drives, Mechatronics 23, 497-504 (2013)

[13] Z. Alexander, C. Ibsen, The politics of airplane production: the emergence of two technological frames in the competition between boeing and airbus, Technology in Society 31, 342-349 (2009)

[14] B. Loechel, J. Goettert, G. Gruetzner, Extreme aspect ratio $\mathrm{NiFe}$ gear wheels for the production of commercially available micro harmonic drive gears, Microsystem Technologies 14, 1675-1681 (2008)

[15] R. Degen, R. Slatter, High speed and low weight micro actuators for high precision assembly applications, Precision Assembly Technologies For Mini and Micro Products 198, 109-117 (2006)

[16] P. Jose, D. Efren, V. Ignacio, Performance of magneticsuperconductor non-contact harmonic drive for cryogenic space applications, Machines 3, 138-156 (2015)
[17] C. Koji, A. Yoshinori, Study on structure for increase of a transmit torque on magnetic harmonic gear with stackable structure, Materials Science Forum 915, 77-82 (2018)

[18] Y. Ren, L. Xu, Analysis on the finite element of electromechanic coupling of flexible gear in electromagnetic harmonic drive, Applied Mechanics and Materials 249-250, 771-777 (2013)

[19] L. Xu, Y. Liang, Output torque for electromagnetic harmonic drive, Advances in Mechanical Engineering 7, $721543(2015)$

[20] L. Xu, Y. Liang, Torque for an electromagnetic harmonic movable tooth drive system, Mechanism and Machine Theory 98, 190-198 (2016)

[21] A. Afanasev, The higher harmonics of the electromagnetic moments of a magnetoelectric reducer, Russian Electrical Engineering 87, 666-671 (2016)

[22] A. Yoshinori, K. Shota, K. Kazuya, Development of magnetic harmonic gear with stackable structure, International Journal of Applied Electromagnetics and Mechanics 52, 809-816 (2016)

[23] C. Koji, K. Takahito, A. Yoshinori, Study on the magnetic harmonic gear with stackable structure - Improvement of its torque density, International Journal of Applied Electromagnetics and Mechanics 59, 629-637 (2019)

[24] R. Liu, J. Tang, Y. Zhang, Air gap magnetic field calculation in ecentric magnetic harmonic gear, Proceedings of 2019 IEEE 2nd International Conference on Electronic Information and Communication Technology, ICEICT 2019, pp. 845-848, January 2019

[25] L. Jing, J. Gong, Research on eccentric magnetic harmonic gear with halbach array, Progress in Electromagnetics Research Letters 89, 37-44 (2020)

[26] G. Fedder, R. Howe, T. Liu, Technologies for cofabricating MEMS and electronics, Proc IEEE 96, 306-322 (2008)

[27] B. Geyer, G. Klimchitskaya, V. Mostepanenko, Analytic approach to the thermal Casimir force between metaland dielectric, Annals of Physics 323, 291-316 (2008)

[28] R. Barauskas, S. Kausinis, H. Tilmans, Investigation of thermo-elastic damping of vibrations of rectangular and ring-shaped mems resonators, Journal of Vibroengineering 11, 177-187 (2009)

Cite this article as: D. Zhao, L. Xu, Y. Fu, Effects of molecule force on free vibration for a micro electromagnetic harmonic drive system, Mechanics \& Industry 22, 12 (2021) 